\title{
Correlations of Neuronal and Microvascular Densities in Murine Cortex Revealed by Direct Counting and Colocalization of Nuclei and Vessels
}

\author{
Philbert S. Tsai, ${ }^{1}$ John P. Kaufhold, ${ }^{4}$ Pablo Blinder, ${ }^{1}$ Beth Friedman, ${ }^{5}$ Patrick J. Drew, ${ }^{1}$ Harvey J. Karten, ${ }^{6}$ \\ Patrick D. Lyden, ${ }^{7}$ and David Kleinfeld ${ }^{1,2,3}$ \\ ${ }^{1}$ Department of Physics, ${ }^{2}$ Center for Neural Circuits and Behavior, and ${ }^{3}$ Graduate Program in Neurosciences, University of California, San Diego, La Jolla, \\ 92093 California, ${ }^{4}$ SAIC Intelligent Systems Division, Arlington, Virginia 22102, Departments of ${ }^{5}$ Pharmacology and ${ }^{6}$ Neuroscience, University of California \\ School of Medicine, San Diego, California 92093, and DDepartment of Neurology, Cedars-Sinai Medical Center, Los Angeles, California 90048
}

It is well known that the density of neurons varies within the adult brain. In neocortex, this includes variations in neuronal density between different lamina as well as between different regions. Yet the concomitant variation of the microvessels is largely uncharted. Here, we present automated histological, imaging, and analysis tools to simultaneously map the locations of all neuronal and nonneuronal nuclei and the centerlines and diameters of all blood vessels within thick slabs of neocortex from mice. Based on total inventory measurements of different cortical regions $\left(\sim 10^{7}\right.$ cells vectorized across brains), these methods revealed: (1) In three dimensions, the mean distance of the center of neuronal somata to the closest microvessel was $15 \mu \mathrm{m}$. (2) Volume samples within lamina of a given region show that the density of microvessels does not match the strong laminar variation in neuronal density. This holds for both agranular and granular cortex. (3) Volume samples in successive radii from the midline to the ventral-lateral edge, where each volume summed the number of cells and microvessels from the pia to the white matter, show a significant correlation between neuronal and microvessel densities. These data show that while neuronal and vascular densities do not track each other on the $100 \mu \mathrm{m}$ scale of cortical lamina, they do track each other on the 1-10 mm scale of the cortical mantle. The absence of a disproportionate density of blood vessels in granular lamina is argued to be consistent with the initial locus of functional brain imaging signals.

\section{Introduction}

The fate of all cells in the cerebral cortex is tied to the cortical vasculature, which supplies oxygen and nutrients, maintains homeostasis, and removes metabolic waste. This dependency is exploited by techniques, such as blood oxygen-level dependent functional magnetic resonance imaging and intrinsic optical imaging, that infer changes in neuronal activity from changes in the local concentration of blood oxygenation (Logothetis et al., 2001). The density and architecture of the vasculature relative to the underlying neurons is thus of central importance for understanding the efficiency and spatial localization of the interaction of brain cells with blood. Yet the structural relationship between cortical vasculature and neuronal tissue, along with details of vascular geometry, remains poorly understood.

One reason for the paucity of anatomical data is the lack of automated methods to collect data from blocks of tissue with

Received July 9, 2009; revised Sept. 9, 2009; accepted Sept. 26, 2009.

This work was supported by the National Institutes of Health (Grants EB003832, MH72570, MH085499, and NS059832 to D.K., NS43300 and NS052565 to P.D.L., and DC006849 to Ravi V. Bellamkonda at the Georgia Institute of Technology) and a Bikura fellowship from the Israeli Science Foundation (to P.B.). We thank Anirvan Ghosh Sebastian H. Seung, Karel Svoboda, and Bruno Weber for useful discussions, the reviewers and editor for critical comments on the original version of this manuscript, and Roger Y. Tsien for the gift of transgenic animals used in Figure 1.

Correspondence should be addressed to David Kleinfeld, Department of Physics 0374, University of California, San Diego, 9500 Gilman Drive, La Jolla, CA 92093-0374. E-mail: dk@physics.ucsd.edu.

DOI:10.1523/JNEUROSCI.3287-09.2009

Copyright $\odot 2009$ Society for Neuroscience $\quad$ 0270-6474/09/2914553-18\$15.00/0 concurrently labeled vasculature and neurons. A second reason is a lack of automated algorithms to vectorize such three-dimensional anatomical datasets. Thus, much of what we know about the relation between neurons and microvasculature is based on estimates obtained from thin samples across a block of tissue using stereological techniques (Russ and Dehoff, 2000). This approach suggests that the known laminar variation of cells in primate visual cortex is not matched by a similar variation in vascular density (Bell and Ball, 1985; Weber et al., 2008; Risser et al., 2009). These estimates, however, do not reveal the detailed spatial arrangements among individual cells and vessels. Nor did past studies consider covariation in cells and microvessels across cortical areas, save for recent exploratory work on small samples of rat cortex (Bjornsson et al., 2008). Finally, although mice are the predominant model for molecular and cellular studies on the mammalian brain, there has been little attention to the relation between neurons and microvasculature in the mouse brain.

In this work we generate and evaluate three-dimensional datasets of cellular and vascular architecture from mouse cortex. We ask: (1) Can we count, in an automated way, all cells and vessels in a thick slab? This mitigates estimation errors associated with counts near the boundary of a region and sampling issues associated with inhomogeneous tissue. (2) Are cell soma uniformly distributed in the space between microvessels? (3) Is there a correlation among the density of neurons and microvessels within a 
column? (4) Is there a correlation among the densities across different cortical areas?

We introduce new histological tools and extend the range of existing tools to address the above questions. All neuronal nuclei, non-neuronal nuclei, and vessels are labeled in millimeter-thick slabs of tissue and imaged with two-photon laser scanning microscopy (TPLSM). The resultant data are processed with automated routines that are optimized for TPLSM data and locate, segment, and classify every soma and blood vessel. Our methods do not assume isotropic variations of cell types, as in stereology, nor do they require uniform intensity of labeling, as in densitometry. Preliminary aspects of this work have appeared (Kaufhold et al., 2007, 2008; Tsai et al., 2007).

\section{Materials and Methods}

\section{Tissue preparation}

Our subjects were 18 National Institutes of Health (NIH) Swiss and C57/BL mice, ranging from 22 to $30 \mathrm{~g}$ in mass $(25 \pm 3 \mathrm{~g}$; mean $\pm \mathrm{SD}$ ), and 2 Sprague Dawley rats, $180 \mathrm{~g}$ in mass; rats were used for the initial development of our procedures. We used transcardial perfusion to label the lumen of the entire vascular network in the brain, as has been demonstrated previously using fluids (Patel, 1983; Cavaglia et al., 2001; Michaloudi et al., 2005), resins (Motti et al., 1986; Harrison et al., 2002; Krucker et al., 2006; Wagner et al., 2006), and gels (Dutly et al., 2006). The basic preparatory procedure for the tissue is summarized as: perfuse animal $\rightarrow$ fill vasculature with fluorescent gel $\rightarrow$ section brain into thick slabs $\rightarrow$ stain all nuclei with organic stain $\rightarrow$ label all neuronal nuclei with antibodies to NeuN $\rightarrow$ match optical index of slab under isovolumetric conditions $\rightarrow$ optically section slab with 3-channel TPLSM.

Our animals were anesthetized with Nembutal, transcardially perfused first with $\sim 60 \mathrm{ml}$ of PBS and then with $\sim 60 \mathrm{ml}$ of $4 \%$ (w/v) paraformaldehyde (PFA) in PBS, all at $0.3 \mathrm{ml} / \mathrm{s}$. This was followed by perfusion with $20 \mathrm{ml}$ of a fluorescent gel perfusate, at $0.6 \mathrm{ml} / \mathrm{s}$, with the body of the mouse tilted by $30^{\circ}$, head down, to ensure that the large surface vessels remained filled with the gel perfusate. The body of the mouse was submerged in ice water, with the heart clamped, to rapidly cool and solidify the gel as the final portion of the gel perfusate was pushed through. The brain was carefully extracted to avoid damage to pial vessels after $\sim 15$ min of cooling (Fig. 1a,b).

\section{Gel composition}

We sought a gel that exhibited low viscosity in the liquid state so that a complete vascular perfusion could be ensured, retained fluorescence after the brain was sectioned, remained stable at room temperature, and was compatible with fixation by PFA. Our gel solution contained porcine skin gelatin type A (no. G1890; Sigma) and fluorescein-labeled-albumin (no. A9771; Sigma). A 2\% (w/v) solution of gelatin is prepared in boiling PBS and allowed to cool to $<50^{\circ} \mathrm{C}$. It is then combined with $1 \%(\mathrm{w} / \mathrm{v})$ fluorescein-albumin, maintained, with stirring, at $40^{\circ} \mathrm{C}$, and filtered through filter paper (no. 1 Whatman) that had been premoistened with PBS. The filtered solution was maintained at $40^{\circ} \mathrm{C}$ before the perfusion.

\section{Assay of the perfusion}

Completeness of the gel perfusion was tested by perfusing transgenic mice that expressed cyan fluorescent protein (FP) and yellow FP in endothelial cells (Tsai et al., 2003). Double labeling of endogenous fluorescent protein and gel perfusate, assayed by visual inspection of planar images obtained with TPLSM from $1 \mathrm{~mm}$ thick sections, showed that all vessels are filled in frontal cortex, parietal cortex (Fig. 1, compare $c, e, g$, $d, f, h)$, thalamus, striatum, and cerebellum ( $100 \%$ of 254 sections across 2 animals). The intensity and pattern of the gel perfusate was unchanged in surface vessels and microvessels for at least 6 months, implying that the labeled albumin was linked to the gel.

\section{Cutting and labeling slabs}

Two orientations of sections were cut, each of which spanned a full hemisphere. One, used for both development of our procedures as well as analysis, was a sagittal section that was cut from the midline to $1.4 \mathrm{~mm}$ lateral of the midline. We examined volumes at anterior-posterior loca- tions $+1 \mathrm{~mm},-1 \mathrm{~mm}$, and $-2 \mathrm{~mm}$ relative to bregma from the face at $1.4 \mathrm{~mm}$ lateral down to $0.8 \mathrm{~mm}$ lateral. The other was a series of coronal sections, centered at bregma $-1 \mathrm{~mm}$ so that one or more sections passed through the vibrissa area. We examined both a volume centered at medial-lateral location $+4 \mathrm{~mm}$ as well as the whole slab. We aligned the brain along the midline and used a razor blade mounted to an optical translation stage (no. 443, Newport) as a means to minimize sample-tosample variability. The slab of tissue was incubated for 10 to $12 \mathrm{~h}$ in a blocking solution of $10 \%(\mathrm{v} / \mathrm{v})$ normal goat serum, $2 \%(\mathrm{v} / \mathrm{v})$ Triton $\mathrm{X}-100$ to improve the permeability of the tissue, and $0.1 \%(\mathrm{w} / \mathrm{v})$ of sodium azide in PBS. This was followed by incubation in a staining solution of $1 \mu \mathrm{g} / \mathrm{ml} \mathrm{4',6-diamidino-2-phenylindole} \mathrm{(DAPI)} \mathrm{dilactate} \mathrm{(no.} \mathrm{D9564,}$ Sigma), a common nuclear stain, and $10 \mu \mathrm{g} / \mathrm{ml}$ of a NeuN-specific antibody that is directly conjugated to the fluorophore Alexa-594 (custom order, Millipore Bioscience Research Reagents). The incubation lasted for $4 \mathrm{~d}$ at room temperature with the goal of complete staining throughout the $1.4 \mathrm{~mm}$ thick slabs. Finally, slabs were washed in PBS.

\section{Isovolumetric index matching}

The maximum imaging depth in formaldehyde-fixed cortical tissue is typically less than that for in vivo tissue (Tsai et al., 2003), and thus our final step was to gradually clear the slab in a sucrose solution. The stained tissue section was equilibrated in a graded manner from $2 \%(\mathrm{v} / \mathrm{v})$ Triton $\mathrm{X}-100$ in PBS to a solution with increasing concentrations of sucrose and $2 \%(\mathrm{v} / \mathrm{v})$ Triton X-100 in PBS over a $6 \mathrm{~h}$ equilibration period per step. The cleared tissue is then mounted on a slide with $60 \%(\mathrm{w} / \mathrm{v})$ sucrose and $1 \%(\mathrm{w} / \mathrm{v})$ agarose gel, and covered with a no. 0 coverslip. The exposed surfaces of the gel are covered with paraffin to prevent dehydration. The lateral face of the sagittal section faces the coverslip so that imaging proceeds from lateral to medial with increasing imaging depth.

Visual inspection of planar images obtained from cleared slabs with TPLSM indicates that the maximum depth for imaging increases with increasing values of the final concentration of sucrose (Fig. $2 a-f$ ), reaching 1.5 $\mathrm{mm}$ for a concentration of $75 \%(\mathrm{w} / \mathrm{v})$ (Fig. $2 g$ ). We chose to use a final concentration of $60 \%(\mathrm{w} / \mathrm{v})$ sucrose for our studies, reached in increments of $15 \%(\mathrm{w} / \mathrm{v})$, over a period of $24 \mathrm{~h}$. This allowed us to image at depths down to 1.0 to $1.2 \mathrm{~mm}$ with excellent clarity of vasculature and nuclei (supplemental Fig. 1, available at www.jneurosci.org as supplemental material).

Solutions that contain high concentrations of sucrose are hyperosmotic with respect to the immersed tissue and thus care must be taken to minimize the shrinkage of tissue. For equilibration with $60 \%(\mathrm{w} / \mathrm{v}) \mathrm{su}-$ crose in the absence of permeabilization with detergent, this can be in excess of $15 \%$ by area (Fig. $2 h$ ). Yet too high a detergent concentration may compromise the integrity of tissue. We revisited this issue and pretreated the fixed tissue with increasing concentrations of Triton X-100. We found that the pretreatment of the tissue with $1 \%(\mathrm{v} / \mathrm{v})$ Triton X-100 reduced tissue shrinkage to $3 \%$ by area at $60 \%(\mathrm{w} / \mathrm{v}$ ) sucrose (Fig. $2 h$ ).

\section{Data acquisition}

We imaged three cortical regions from the sagittal section, centered at 2 mm caudal of bregma, $1 \mathrm{~mm}$ caudal of bregma, and $1 \mathrm{~mm}$ rostral of bregma (Fig. 3a), and the entire cortical area of the coronal sections, with a custom two-photon microscope (Tsai et al., 2003; Tsai and Kleinfeld, 2009). Hardware control and data acquisition were performed using the MPScope software suite (Nguyen et al., 2006; Nguyen et al., 2009). Images were taken through a $40 \times$ magnification IR Zeiss dipping objective [0.8 numerical aperture (NA)] and acquired at 5 frames-per-second with an averaging of 2 to 4 frames per axial position. Each frame consists of $256 \times 256$ voxels at $1 \mu \mathrm{m}$ per voxel. Image stacks consist of 700 frames, separated axially by $1 \mu \mathrm{m}$, and neighboring stacks were taken with an overlap of $56 \mu \mathrm{m}$. An image dataset consists of between 7 and 9 image stacks that collectively span, on average, $256 \mu \mathrm{m}$ by $1656 \mu \mathrm{m}$ by $700 \mu \mathrm{m}$ or $0.3 \mathrm{~mm}^{3}$ of tissue. Thus each dataset consists of three 300 megavoxel files.

The tissue sections contain cell nuclei labeled with DAPI, vessels filled with fluorescein labeled gel, and neuronal nuclei labeled with Alexa-594 conjugated to $\alpha$-NeuN. The excitation wavelength was $800 \mathrm{~nm}$. All emitted light was filtered through a short pass interference filter (E680SP, Chroma) to block excitation light and then split into 3 channels for simultaneous collection (Fig. $3 b$ ). For the Violet channel, emitted light is 

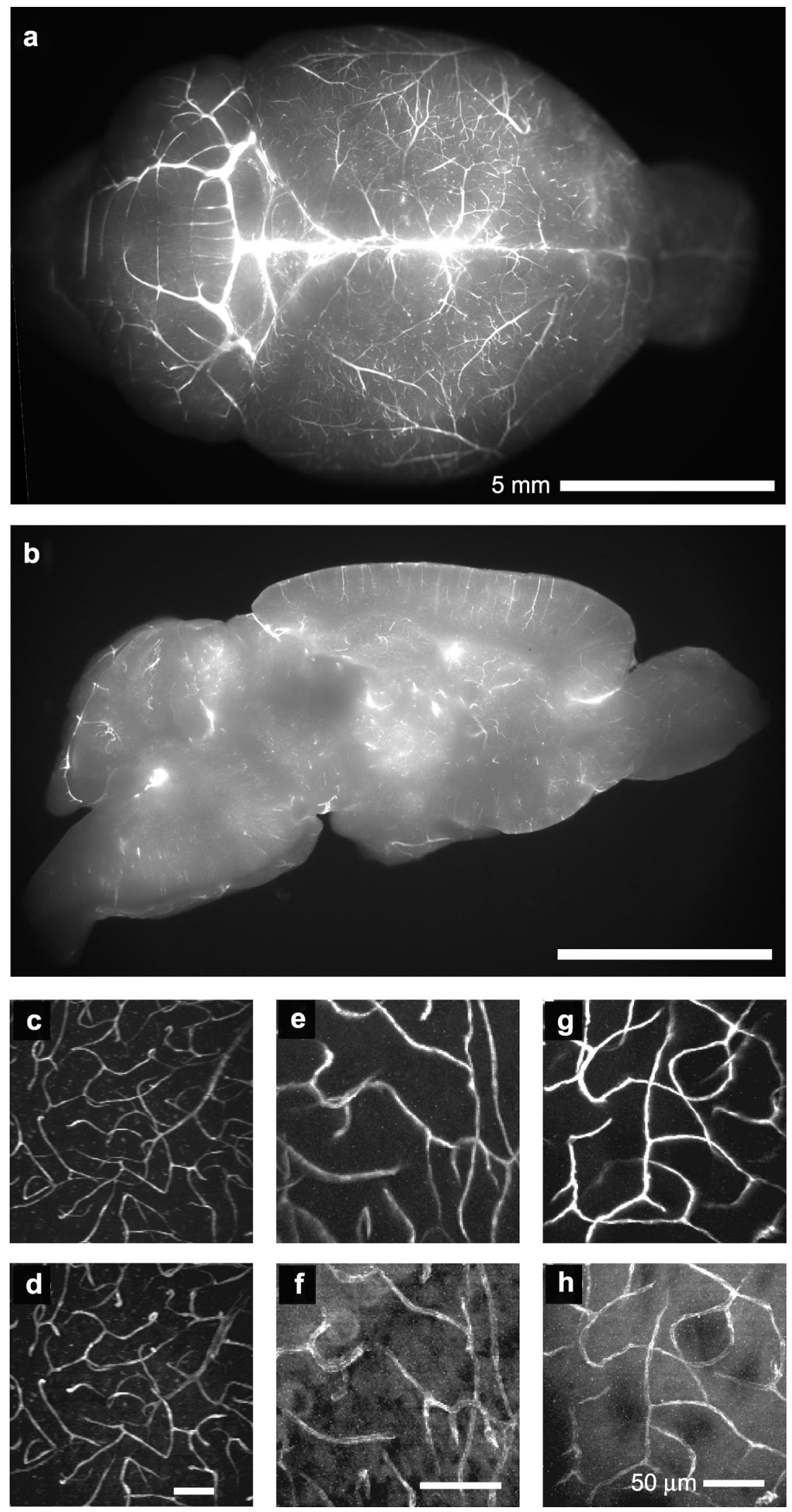

Figure 1. Perfusion of brain vasculature with a fluorescent gel-based perfusate in comparison with a label of endothelial cells. We visualize the brain vasculature in a NIH Swiss mouse. $\boldsymbol{a}$, Wide-field epi-fluorescence photograph montage of a NIH Swiss mouse brain perfused with a fluorescein-conjugated gel. Both veins and arteries are visibly labeled, although some surface vessels, particularly those that were immediately contiguous to the central sinus, were lost during the brain extraction process. $\boldsymbol{b}$, Widefield epi-fluorescence photograph of a sagittal section from a brain perfused with fluorescein-conjugated gel. $\boldsymbol{c}-\boldsymbol{h}$, Evidence of reflected from a dichroic mirror centered at $505 \mathrm{~nm}$ (550 DCLP; Omega Optical) and bandpass filtered through an interference filter centered at $450 \mathrm{~nm}$ (450DF50, Omega Optical) and $2 \mathrm{~mm}$ of colored glass (BG30, Andover). For the Green channel, emitted light is transmitted through the dichroic centered at 550 $\mathrm{nm}$, reflected from a dichroic centered at 550 nm (550 DRLP, Omega Optical), and bandpass filtered through an interference filter centered at $535 \mathrm{~nm}$ (535DF25, Omega Optical) and 2 $\mathrm{mm}$ of colored glass (BG30). For the Red channel, emitted light is transmitted through the dichroic centered at $505 \mathrm{~nm}$, transmitted through the dichroic centered at $550 \mathrm{~nm}$, and bandpass filtered through the combined action of a second E680SP filter and $1 \mathrm{~mm}$ of colored glass (OG590, Andover). Last, two calibration images are taken for each spectral channel at each imaging session. The first calibration file is an image with the excitation laser blocked, while the second is an image of a uniform fluorescent bath. Under our imaging conditions there was negligible photobleaching of any of the dyes.

The point spread function (PSF) of our microscope was determined by imaging $0.2 \mu \mathrm{m}$ diameter fluorescent beads (Polysciences). Near the surface, the PSF was well described by a three-dimensional Gaussian with full-width at half-maximum of $0.4 \mu \mathrm{m}$ in along the $x$ - and $y$-axes and $2 \mu \mathrm{m}$ along the $z$-axis for the $40 \times$ magnification dipping objective. The data we collect is under-sampled along the $x$ - and $y$-axes and oversampled along the $z$-axis.

\section{Data analysis}

The overall plan of analysis is to transform raw image stacks into four overlapping volumetric files that contain: (1) the coordinates of every cell nucleus; (2) the classification of every nucleus as neuron or non-neuron; (3) the coordinates of the centerline of every vessel, along with the diameter with respect to the centerline; and (4) the assignment of contiguous vessels as microvessels. These processes are summarized in the flow diagram of Figure 4 and are implemented with a series of algorithms written in the Matlab programming language and denoted the Volumetric Image Data Analysis (VIDA) suite (supplemental Material, available at www.jneurosci.org); the VIDA suite is further available at http://physics.ucsd.edu/neurophysics/ links.html.

\section{Preprocessing}

These steps correct for the bending of light at the buffer-to-tissue interface, nonuniform flu-

\section{$\leftarrow$}

completeness of the gel fill. Maximal projections from TPLSM image stacks $>50 \mu \mathrm{m}$ of depth taken from the brains of transgenic mice with CFP-expressing endothelial cells after perfusion with fluorescein-conjugated gel. The top row shows the fluorescein label in neocortex (c), cerebellum (e), and striatum $(\boldsymbol{g})$. The bottom row shows the (FP transgenic label in the same fields-of-view $(\boldsymbol{d}, \boldsymbol{f}, \boldsymbol{h})$. All vessels expressing CFP also contain fluorescein-conjugated gel perfusate, and vice versa. Note that in the cerebellum (f), CFP is also expressed within the Purkinje cells (Tsai et al., 2003). 
orescence across the field (Tsai and Kleinfeld, 2009) and demix the contributions of fluorescence from each of the three stains to anatomically relevant channels (Fig. $3 b-h$ ).

Focus-depth correction. The optical index of brain is $n_{\mathrm{b}} \approx 1.42$ (Stepnoski et al., 1991), compared with $n_{\mathrm{W}}=1.33$ for water. This results in a shift in the apparent depth of the focus, in addition to spherical aberration. The focus moves by $n_{\mathrm{b}} / n_{\mathrm{W}}=1.06 \times$ the distance that the objective moves near the optical axis but by $\tan \left[\sin ^{-1}\left(\mathrm{NA} / n_{\mathrm{W}}\right)\right] / \tan \left[\sin ^{-1}\left(\mathrm{NA} / n_{\mathrm{b}}\right)\right]=$ $1.10 \times$ the distance that the objective moves for the marginal rays of our NA $=0.8$ dipping objective. We correct the scaling of the $z$-axis by the mean of these two values, so that the focus moves by $1.08 \mu \mathrm{m}$ when the objective is lowered by $1.00 \mu \mathrm{m}$.

Dark-level correction. The mode of the intensity of the dark calibration image for the Violet, Green, and Red channels is subtracted from all voxels in the respective channel to correct for offsets in the detector electronics.

Field normalization. We correct for uneven illumination, as occurs when both $x$-axis and $y$-axis scan mirrors are not focused at the back aperture of the objective, and uneven detection, as is caused by vignetting (Tsai and Kleinfeld, 2009). Images from a fluorescent bath calibration image for the Violet, Green, and Red channels are averaged and smoothed and then used to normalize the signals in respective channels via voxelwise division (Fig. $3 c-e$ ).

Primary spectral demixing. Demixing corrects for spectral cross talk between imaging channels. While contamination by the long tail of lower-wavelength fluorophores into higher-wavelength channels is unavoidable, the spectral windows for imaging were chosen so that higher-wavelength fluorophores did not significantly contaminate lower-wavelength collection windows. Spectral demixing then reduces to channel subtraction with an appropriate weighting factor. Determination of the weighting factors is determined automatically by equalizing the mode of the values of voxels in regions inside and outside a delimiting mask. In the first of two rounds of demixing, the mask is taken as a user-defined small percentage of the brightest pixels in the contaminating channel. The median of the weighting factors calculated for all image stacks is applied to spectrally demix all image stacks. The three demixed channels of volumetric grayscale data are denoted as the DAPI, fluorescein, and Alexa-594 channels (Fig. $3 f-h$ ).

File montaging. A correlation-based algorithm is used to align the overlapping region of neighboring image stacks and form a single three-dimensional montage. To significantly reduce processing time, sliding correlations are performed on the maximal projections of the overlapping regions taken at three orthogonal angles. The proper location of the image stack within the montage is associated with the position of the maximal correlation. Correlations are performed with the vessel channel, since it has the sparsest labeling, and the coordinates are applied to all channels.

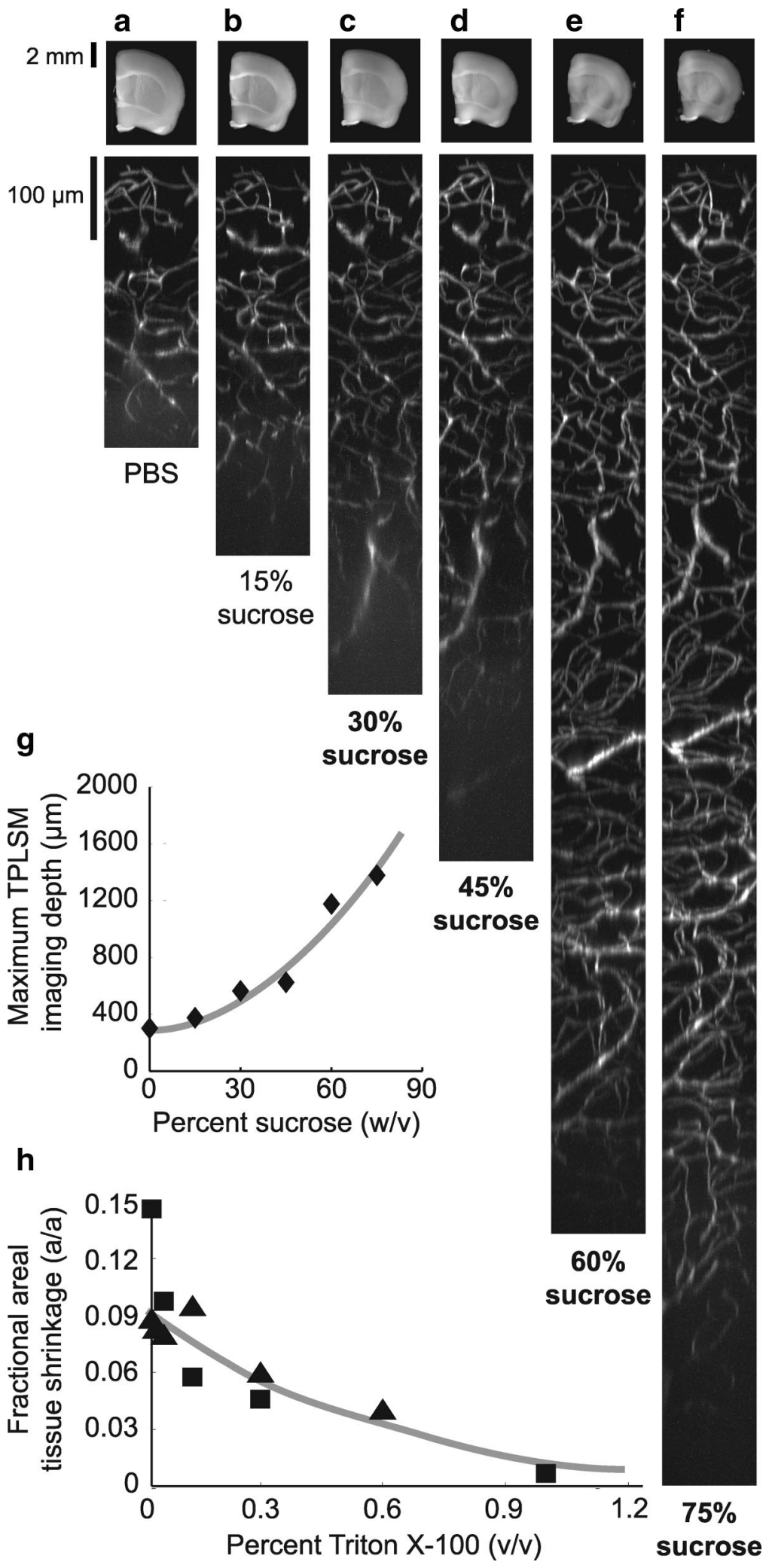

Figure 2. Tissue clearing for TPLSM with sucrose and Triton X-100 immersion to increase imaging depth with minimal tissue shrinkage. All data are from Sprague Dawley rats. $\boldsymbol{a}-\boldsymbol{f}$, Maximal projections in $x-z$ generated from TPLSM image stacks taken in cortex of the same $2 \mathrm{~mm}$ thick coronal tissue section after immersion in increasing concentrations of sucrose in PBS. Lowmagnification photographs of the tissue section after each immersion step are shown above the TPLSM projection. $\boldsymbol{g}$, Summary of the maximum TPLSM imaging depth versus concentration of sucrose for the tissue shown in panels $\boldsymbol{a}$ to $\boldsymbol{f}$; the curve is solely to guide the eye. $\boldsymbol{h}$, Summary plot of the reduction in tissue shrinkage in a 1-mm-thick coronal section versus concentration of Triton $\mathrm{X}-100$. Squares and triangles represent two different datasets. The curve is solely to guide the eye. 


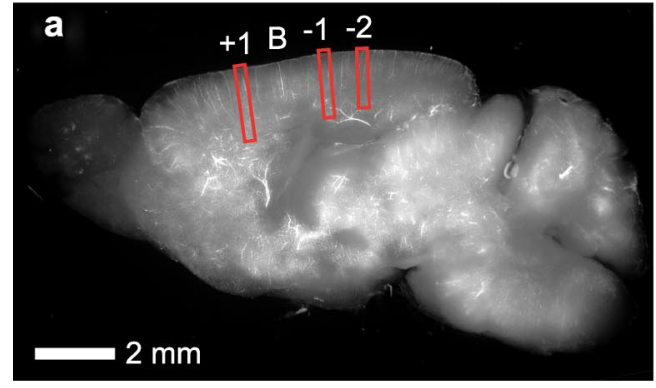

Raw image data

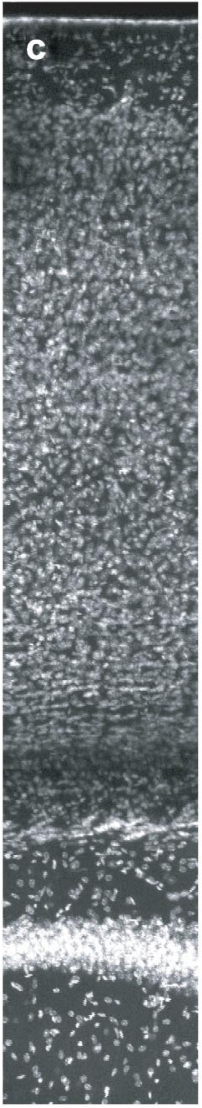

Violet

channel
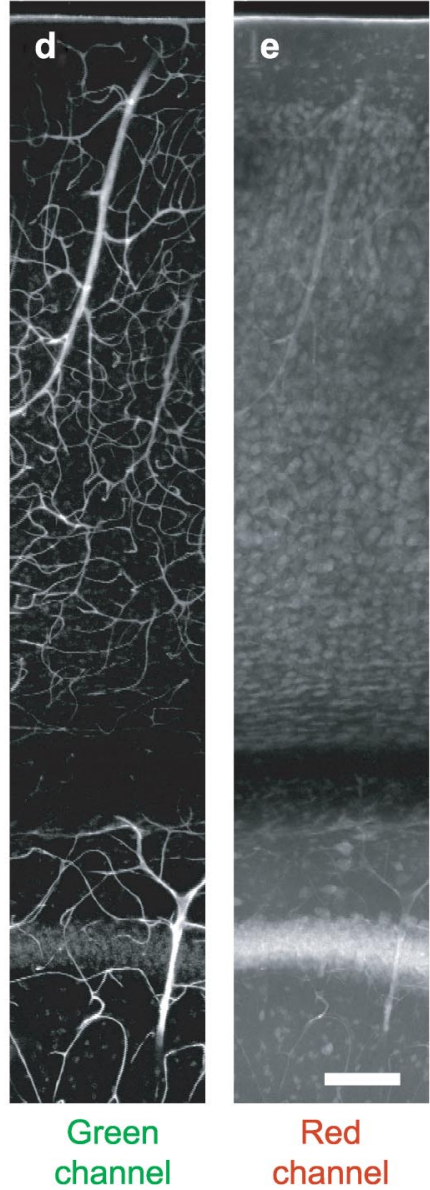

Red

channel

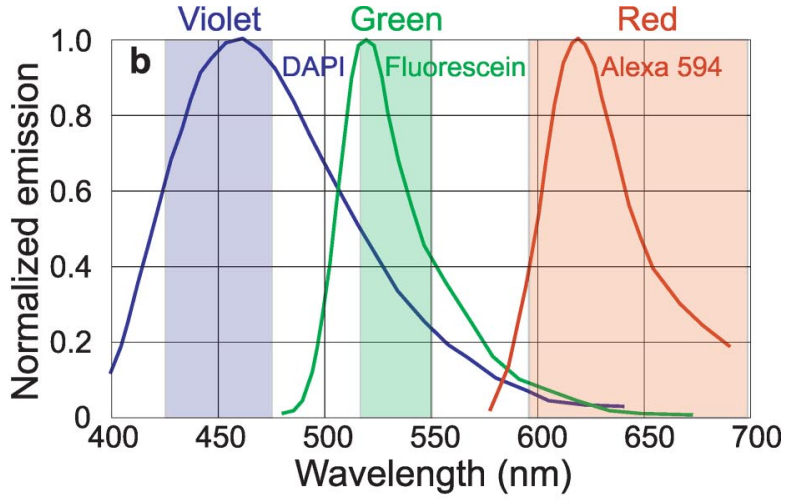

Demixed image data

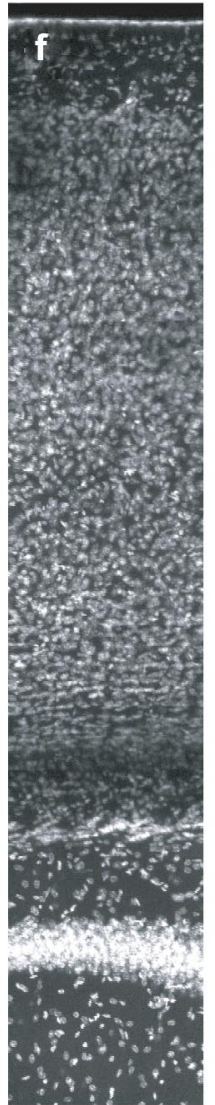

DAPI

(nuclei)

channel

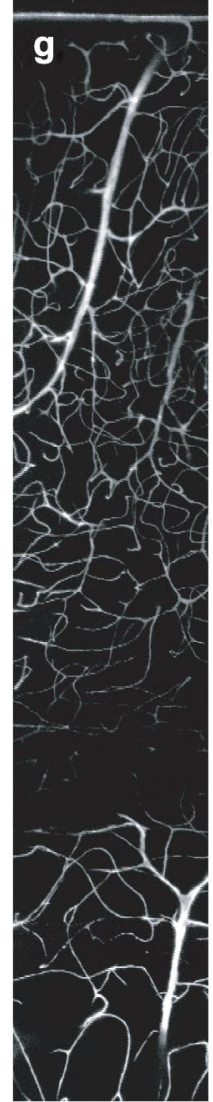

Fluorescein

(vasculature) channel

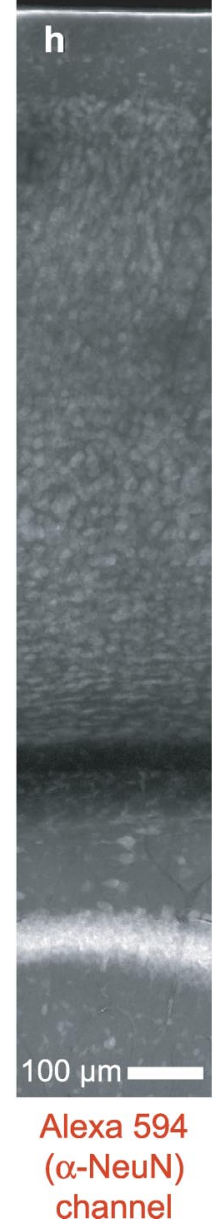

Figure 3. Three-channel TPLSM vascular and cell nuclei imaging from three regions of mouse cortex at a depth of $300 \mu \mathrm{m}$. Sagittal sections from a NIH Swiss mouse, $1.4 \mathrm{~mm}$ thick, were imaged at three rostral-caudal positions. $\boldsymbol{a}$, An epi-fluorescence micrograph of the sagittal section. The three rectangles indicate three regions of interest centered at bregma $+1 \mathrm{~mm}$, bregma $-1 \mathrm{~mm}$, and bregma $-2 \mathrm{~mm}$, that span the depth of cortex. $\boldsymbol{b}$, Emission spectra for the nuclear stain DAPI, the vascular stain fluorescein, and the neuronal nuclear label Alexa 594 , along with the collection bands for emitted light in the violet, green, and red. $c-e$, Maximal projection of TPLSM image data from the bregma -2 mm region before spectral demixing. While the violet spectral window (c) contains predominantly DAPI signal, the green spectral window (d) contains both fluorescein and DAPI signal components. Similarly, the red spectral window (e) contains contributions from Alexa 594, fluorescein, and DAPI. $\boldsymbol{f}-\boldsymbol{h}$, The same data as shown in $\boldsymbol{c}-\boldsymbol{e}$ after spectral demixing.

Data sub-blocking. The montaged datasets are sufficiently large that differences in staining intensity and in signal-to-noise ratios are present across the montage. Each montage was thus divided into overlapping sub-blocks for segmentation and further demixing. These operations were performed with parameters, such as threshold levels, calculated separately for each subblock. We used sub-blocks of $\sim 200 \times 150 \times 150$ or 5 megavoxels each.

\section{Nuclear segmentation}

The VIDA suite is used to calculate the coordinates of every cell nucleus within the tissue volume based on DAPI staining.
Cell nuclei. Cell nuclei segmentation is achieved by spatial bandpass filtering of the DAPI channel (Fig. 5a). Each sub-block is high-pass filtered by subtracting a low-pass filtered version of the sub-block, computed by filtering the sub-block with an isotropic three-dimensional Gaussian with $\mathrm{SD} \sigma_{\text {cell-bkg }}$, from the original data. We chose $\sigma_{\text {cell-bkg }}=$ 4.5 pixels to be comparable to the largest cell size. Next, the high-pass filtered sub-block was convolved with an isotropic three-dimensional Gaussian of SD $\sigma_{\text {cell-local }}$ to produce a band-filtered cell sub-block (Fig. $5 b)$. We chose $\sigma_{\text {cell-local }}=2$ pixels, which corresponds to a full-width at 
half-maximum of $4.7 \mu \mathrm{m}$, to be large compared to intranuclear structures but small compared to typical internuclear separation.

A binary cell mask was generated by thresholding each filtered cell sub-block against a value automatically selected to be much higher than fluctuations in the intensity of the background (Fig. $5 c$ ). To determine the threshold values, $T_{\text {cell }}$, we first compute the local maxima of the intensity within each bandpass filtered cell sub-block. A histogram of the local maxima shows two peaks. A sharp peak at low values of intensity corresponds to fluctuations in background regions; the mode of this peak is denoted $M_{\text {peak, }}$ and the SD of this peak, found by fitting to values less than the mode, is denoted $\sigma_{\text {peak }}$. A broad peak at relatively high intensities corresponds to labeled nuclei. The threshold is set at $T_{\text {cell }}=M_{\text {peak }}+z \sigma_{\text {peak }}$ to isolate this peak, where the parameter $z$ was empirically set to $z=5$. The mask is formed by setting all values in the sub-block that are greater than $T_{\text {cell }}$ to one and all other values to zero. Connected volumes with $<100$ voxels are removed to eliminate noise volumes. This results in a set of isolated components that represent single or overlapping nuclei (Fig. 5e). The volume of the resulting cell mask is sensitive to the choice of $z$, while the location and number of cell centroids computed from the masks are relatively insensitive to the choice of $z$.

Cell centroids. The location of cell centroids were determined from the binary cell mask using morphological operations. First, a threedimensional ultimate erosion was performed on the binary mask (Fig. 5c). This results in singular retained voxels for isolated components that are roughly spherical in shape (Fig. $5 d$ ). Merged spherical components that meet as a double-bubble result in two separated voxel islands. Isolated ellipsoidal components can result in a line of connected or closely spaced voxels, as will merged components that are not convex at their junction. This operation results in a list of candidate centroid locations that are subsequently thinned via simple point removal to a single voxel location per contiguous set of voxels associated with a given centroid.

We tested for centroids that were spaced closer than a value $D_{\text {cell_min }}$, with the assumption that such centroids are part of the same nucleus. Thus when only a pair centroids were within $D_{\text {cell min }}$, one was eliminated at random. When groups of three or more centroids were within a distance $D_{\text {cell_min }}$ of each other, we successively eliminated centroids until no two centroids were located within a distance $D_{\text {cell_min }}$ of each other (Fig. $5 e$ ). We chose the empirical value $D_{\text {cell_min }}=5$ voxels $=5 \mu \mathrm{m}$; larger values led to the elimination of cells. This operation results in a list of locations of putative nuclei.

\section{Vascular segmentation}

The VIDA suite is used to obtain a volumetrically correct binary mask of all vasculature based on the fluorescein fills. The sub-blocks from the fluorescein channel form the vessel grayscale volume (Fig. 6a). Vascular segmentation follows a multistep process and includes the use of matched filters (Fig. $6 b-m$ ).

vascular mask

sub-blocks

Microvessel

mask sub-blocks
Three channel

raw image stacks
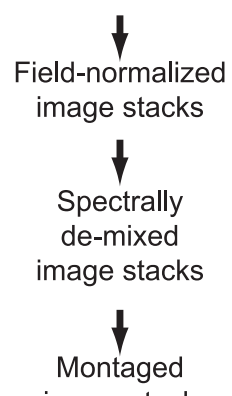

image stack

$\downarrow$

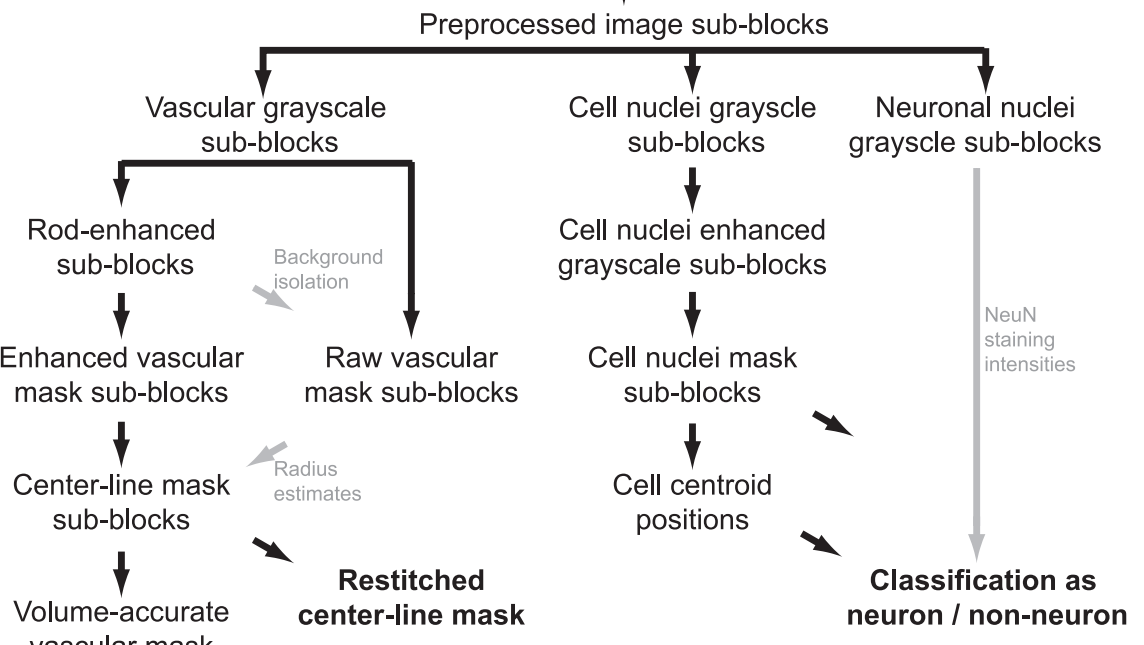

Restitched vascular mask

\section{Restitched microvessel mask}

Figure 4. Process flow diagram for the analysis of cellular and vascular volumetric data. Three-channel data are normalized, spectrally demixed, montaged, and then divided up into small local three-dimensional sub-blocks. Each sub-block is processed independently. Vascular processing begins by matched filtering to 82 rods at various orientations to produce a "Rod-enhanced sub-block" (detailed in Fig. 6). Local filtering and variable local thresholding is applied to produce a "Raw vascular mask" that maintains appropriate vessel diameters, and a connectivity-preserved "Enhanced vascular mask." Connectivity-conserved thinning produces a monofilament "Centerline mask." Voxel-by-voxel evaluation of each center-line point in the yields a true vessel radius based on the local intensity pattern in the "Vascular grayscale sub-blocks." Isotropic, volume-accurate masks are reconstructed from the centerline and radius data by successive placement of overlapping spheres. Voxel-by-voxel radius selection is used to categorize vessel regions by size. Finally, the various sub-blocks are restitched to produce a montaged mask of the full dataset. Cell nuclei data processing begins with matched filtering to a spherically symmetric kernel to produce a "Cell nuclei enhanced grayscale sub-block." The "Cell nuclei enhanced grayscale sub-blocks" are automatically thresholded (detailed in Fig. 5) to produce "Cell nuclei masks" from which cell centroids are computed and concatenated. Finally, using the centroids and cell masks from the cell nuclei data, local evaluation of the signal in the corresponding pixels of the neuron nuclei grayscale sub-blocks leads to classification of each cell nuclei as neuronal or non-neuronal (detailed in Fig. 8).

Secondary spectral demixing 1. An additional round of demixing makes use of the results of the nuclei segmentation. The binary cell mask (Fig. $5 c$ ) is used as to refine the spectral demixing coefficient for removal of fluorescent signals from cell nuclei that still contaminate the vascular data.

Rod filtering. The vessel grayscale volume (Fig. $6 a$ ) in each vascular subblock is matched-filtered with rods of length 11 voxels at 82 different orientations uniformly spaced over the half-sphere. The voxel-by-voxel maximal response to the set of 82 filters is retained to produce a vessel-enhanced grayscale volume (Fig. $6 a-c$ ). The maximum value of this volume is denoted $I_{\max }^{\text {filt }}$

Raw vascular mask. A binary mask of the vasculature is produced from the un-filtered vessel grayscale volume (Fig. $6 a$ ) as an intermediate step 


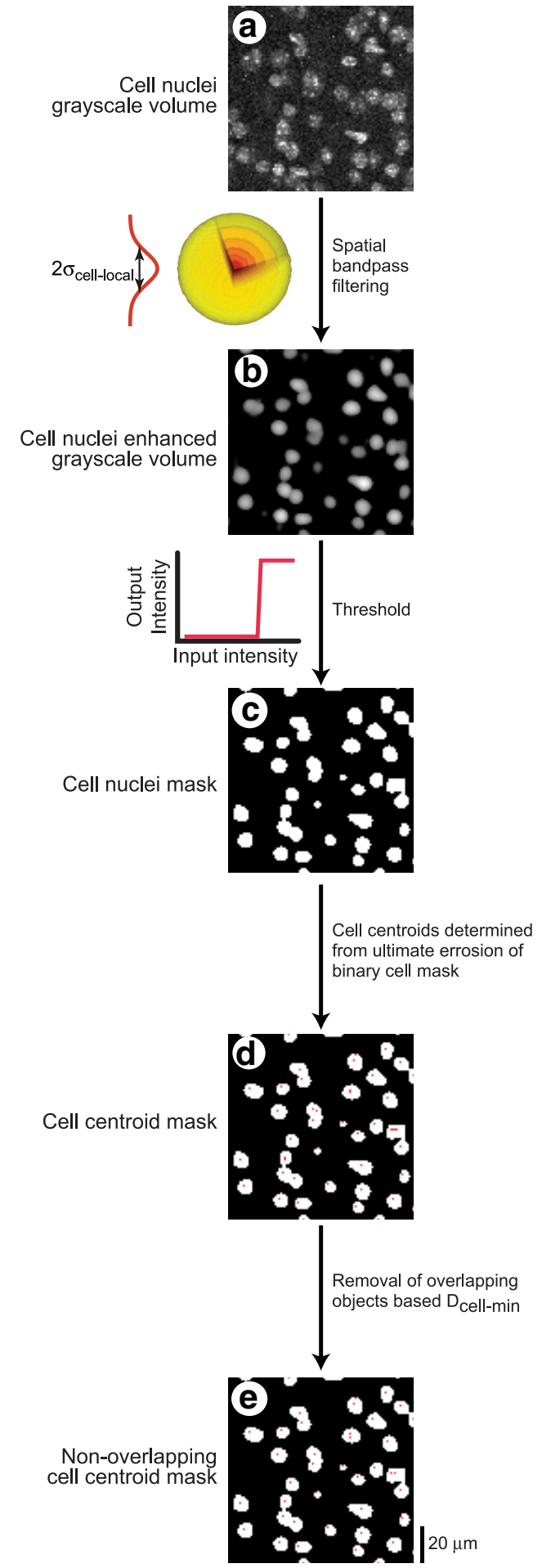

Figure 5. Process flow diagram for automated identification and segmentation of all cell nuclei. $\boldsymbol{a}, \boldsymbol{b}$, Cell nuclei data processing begins with matched filtering of the "Cell nuclei grayscale volume" $(\boldsymbol{a})$ with a spherically symmetric kernel to produce a "Cell nuclei enhanced grayscale volume" $(\boldsymbol{b})$. c, The locus of local intensity maxima in the "Cell nuclei enhanced grayscale volume" ( $\boldsymbol{b}$ ) are determined, their corresponding intensities are compiled, and a threshold is set at a $z$-score of 5 for the distribution of peak intensity values. This threshold is applied to the "Cell nuclei enhanced grayscale volume" (b); connected volumes with $<100$ voxels are eliminated. The result is a "Cell nuclei mask." $\boldsymbol{d}$, Application of an ultimate erosion process to the "Cell nuclei mask" (c) results in a "Cell centroid mask," from which a list of cell centroid locations is compiled. $\boldsymbol{e}$, A minimal cell diameter is used to merge multiple centroid points within the same cell and form the "Nonoverlapping cell centroid mask." toward estimating both the intensity of background regions and the radii of the vessels. We first estimate the background regions from the vessel enhanced grayscale volume (Fig. $6 c$ ). A preliminary binary threshold is empirically set as one SD above the mean value of the intensity for the quartile of voxels with the lowest intensity in the vessel enhanced grayscale volume (Fig. 6 c). This mask is dilated until it occupies $80 \%$ of the total volume, inverted, and used to extract the $20 \%$ of voxels that are furthest from any vessel. These are designated background voxels. These voxels are then evaluated for the unfiltered grayscale data (Fig. $6 a$ ); the mode of their intensity is denoted $l_{\text {back }}^{\text {unfilt }}$ and the SD of their intensity is denoted $\sigma_{\text {back }}^{\text {unfilt }}$.

As a means to convert the vessel grayscale volume (Fig. $6 a$ ) to a binary mask, we first generate a threshold value for each voxel. We form two copies of the volumetric data, low-pass filter one copy with a Gaussian of width (1/e) 11 voxels, and low-pass filter the other copy with a Gaussian of width 3 voxels. For every voxel, the threshold is chosen as $1.5 \times$ the lower of the two values from the low-pass filtered datasets. The values of these thresholds are clamped at an upper bound of $0.15 I_{\max }^{\text {filt }}$ and a lower bound of $I_{\text {back }}^{\text {unfilt }}+4.4 \sigma_{\text {back }}^{\text {unfilt }}$; recall that $\operatorname{erf}\{4.4\}=0.005$. Application of this voxel-by-voxel procedure results in a binary vascular mask. The use of two low-pass filters in determining the threshold preserves small vessels with weak fluorescence that are located proximal to large, bright vessels. Furthermore, this procedure results in a binary mask of the vasculature that has approximately accurate diameters, but may include small artifactual gaps along vessels of low intensity.

As final steps, connected components in the mask with $<27$ contiguous voxels are removed, and the mask is made convex by a morphological closing operation that consists of 3 iterations of constrained filtering. The result is denoted the raw vascular mask (Fig. 6i).

Enhanced vascular mask. A dilated binary mask of the vasculature is produced from the filtered vessel grayscale volume (Fig. $6 c$ ). We estimate background regions by dilating the raw vascular mask (Fig. 6i) until it occupies $80 \%$ of the total volume. This mask is then inverted and used to extract the $20 \%$ of voxels in the vessel-enhanced grayscale volume (Fig. $6 c$ ) that are furthest from any vessel. These are designated background pixels; the mode of their intensity is denoted $I_{\text {back }}^{\text {filt }}$ and the SD of their intensity is denoted $\sigma_{\text {back. }}^{\text {filt }}$.

As a means to convert the vessel enhanced grayscale volume (Fig. $6 c$ ) to a binary mask, we first generate a threshold value for each voxel. We form two copies of the volumetric data, low-pass filter one copy with a Gaussian of width (1/e) 21 voxels, and low-pass filter the other copy with a Gaussian of width 7 voxels. For every voxel, the threshold is chosen as $1.5 \times$ the lower of the two values from the low-pass filtered datasets. The values of these thresholds are clamped at an upper bound of $0.10 I_{\max }^{\text {filt }}$ and a lower bound of $I_{\text {back }}^{\text {filt }}+4.4 \sigma_{\text {back }}^{\text {filt }}$. Application of this voxel-by-voxel procedure results in the binary enhanced vascular mask. This procedure results in a binary mask of the vasculature that overestimates the diameters, and admits only a few small gaps along vessels of low intensity.

As final steps, connected components in the mask with $<27$ contiguous voxels are removed, and the mask is made convex by a morphological closing operation that consists of 3 iterations of constrained filtering. The results is denoted the enhanced vascular mask (Fig. $6 d$ ).

\section{Vascular center-lines}

We use the above masks to determine the center-lines of the vessels by thinning the enhanced vascular mask (Fig. $6 d$ ). This process involves multiple steps and intermediate results (Fig. $6 e-m$ ). We consider first the creation of three auxiliary maps, each of which makes use of a Euclidean distance transformation of a corresponding vascular mask. Entries in the map report the distance from each active voxel, i.e., with values of 1 , to the nearest inactive voxel, i.e., with a value of zero. For example, a vessel that lies in the horizontal plane with a width of 6 voxels has the sequence 001233210 while one with a width of 7 voxels has the sequence $\bullet \bullet 012343210 \bullet \bullet \bullet$.

Approximate radius map. This map is used to approximate the diameter of each vessel. This is determined by taking a Euclidean distance transform of the raw data mask (Fig. 6i), to form an approximate radius map of each vessel (Fig. 6j).

Removal sequence map. This map is used to differentiate among voxels with the same value in a Euclidean distance transform map based on whether or not they are near the edge of a vessel. We generate a Euclidean 
distance transform map of the enhanced vascular mask (Fig. 6d). We then copy this mask, spatially low-pass filter the copy using a $3 \times$ $3 \times 3$ voxel block, multiply the filtered copy by a number much smaller than the quantization of distances, e.g., $1 \times 10^{-6}$, and then subtract the copy from the original distance transformed map. The result is a removal sequence map (Fig. 6k) with a gradient of Euclidean distances that, locally, points outward.

Vascular centering map 1. This map is used to remove web-like loops from the thinned data during recentering of the mask. It is generated by taking a Euclidean distance transform of the enhanced vascular mask (Fig. 6d). The resulting map is generally elongated along the $z$-axis by the anisotropic PSF which results in multiple maxima along the $z$-axis. This leads to an ambiguity as to the center of the vessel. To resolve this, we perform multiple iterations of spatial filtering, using a $3 \times 3 \times 3$ voxel block, and masking of the enhanced vascular mask (Fig. $6 d$ ). The number of iterations is taken as $1.5 \times$ the largest value across the approximate radius mask (Fig. 6j). The result is a vascular centering map (Fig. 6l) in which one voxel in each given cross-section has the largest value.

Thinning. The thick vessels in the enhanced vascular mask (Fig. $6 d$ ) are thinned to singlevoxel lines along their center to define a centerline mask. Our algorithm was designed to minimize the progressive trimming of free-ends. In the first pass of thinning, a connectivitymaintaining algorithm was applied to the enhanced mask, i.e., simple point removal or distance-ordered homotopic thinning. We used the removal sequence map as a guide and, starting with the lowest values, removed a voxel so long as the removal did not reduce the connectivity on a $3 \times 3 \times 3$ neighborhood about the voxels. By construction, the removal process starts on the outside of a vessel and moves inward toward larger distance vales. The removal process among shells of voxels with the same distance value is from inside to outside. The end product is a thinned mask with preserved connectivity (Fig. 6e). Note that, as a practical matter in determining connectivity, the analysis for all $2^{33}-1 \sim 7 \times 10^{7}$ possible neighborhood configurations is stored as a lookup table.

Recentering. The local inside-to-outside aspect of the voxel removal process results can produce loops of voxels that are connected to the centerline and form a webbed structure. These loops are eliminated by a process of recentering. We analyze the local $3 \times 3 \times 3$ voxel neighborhood of each active voxel that is not at an end-point in the centerline mask (Fig. 6c). Loops are removed by shifting non-end-point voxels in three-step hierarchical sequence: (1)

A voxel is removed if doing so does not change the local connectivity. (2) If removal is not possible, we move the voxel to an unoccupied location that must be within one pixel, must have a larger value in centering map 1 , and must not change the local connectivity. (3) If both removal and movement of a voxel is not possible, but there is an unoccupied neighboring voxel with a larger value in centering map 1, we activate that voxel. Steps 1 to 3 are repeated for all center-line voxels and iterated until no further changes occur, to form the recentered mask (Fig. 6f).

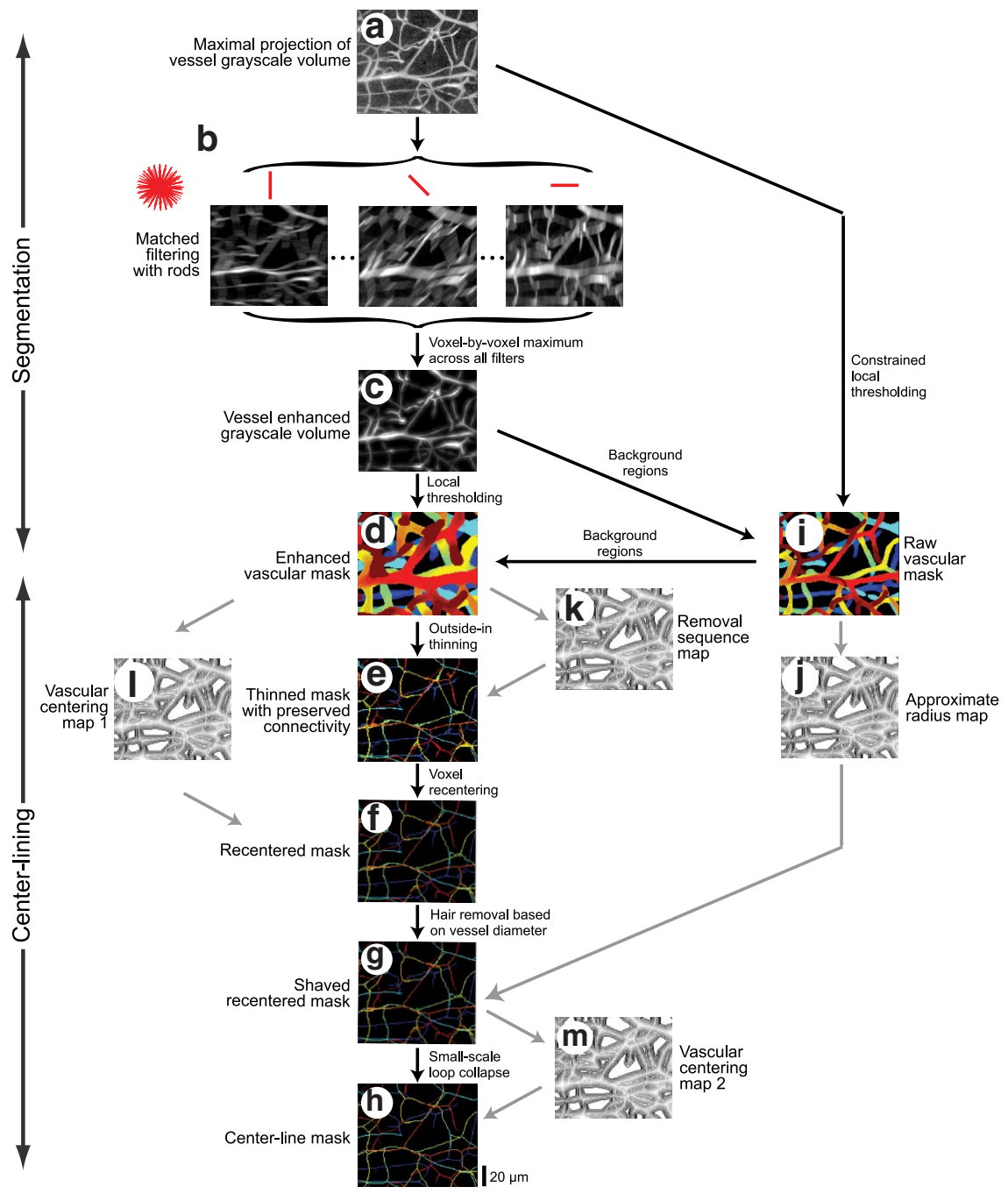

Figure 6. Process flow diagram for automated identification and segmentation of vascular subvolumes. $\boldsymbol{a}, \boldsymbol{b}$, Processing begins by matched filtering of the "Vessel grayscale volume sub-block" (a) to 82 rods at various orientations in three-dimensions, yielding 82 rod filtered volumes $(\boldsymbol{b})$. $\boldsymbol{c}$, The voxel-by-voxel maximal response across all rod filtered volumes $(\boldsymbol{b})$ is retained to produce a "Vessel enhanced grayscale volume." $\boldsymbol{d}$, Local filtering and a local variable threshold is applied to the "Vessel enhanced grayscale volume" (c) to produce an "Enhanced vascular mask." This mask overestimates vascular diameters, but reduces the occurrence of small gaps in the vasculature. $\boldsymbol{e}$, A connectivity-conserving thinning process is applied to the "Enhanced vascular mask" (c) based on the auxiliary "Removal sequence map" ( $\boldsymbol{k}$; defined below). This produces a "Thinned mask with preserved connectivity" that may contain webbed structures of small loops and hairs. $\boldsymbol{f}$, A recentering process is applied to the "Thinned mask" (e) based on the auxiliary "Vascular centering map 1" (I; defined below) to collapse the webbed structures and results in a "Recentered mask." $\boldsymbol{g}$, Short hairs are removed from the "Recentered mask" (f) based on the auxiliary "Approximate radius map" ( $\boldsymbol{k}$; defined below) to produce a "Shaved recentered mask." $\boldsymbol{h}$, The recentering process is repeated on the "Shaved recentered mask" $(\boldsymbol{g})$ based on the auxiliary "Vascular centering map 2" ( $\boldsymbol{m}$; defined below) to collapse small loops in the mask, and results in a monofilament "Center-line mask" of the vasculature. We now turn to auxiliary calculations. $i$, An auxiliary mask is generated by local filtering and a local variable thresholding of the "Vessel grayscale volume" to produce the "Raw vascular mask." This mask contains approximately accurate diameters, but may contain small gaps along vessels of low signal-to-noise. $\boldsymbol{j}-\boldsymbol{m}$, Three auxiliary maps are generated by filtering the Euclidean distance transforms of specific masks. The "Approximate radius mask" (i) is derived from the "Raw vascular map" (i). The "Removal sequence map" $(\boldsymbol{k})$ and the "Vascular centering map 1" (I) are derived from the "Enhanced vascular mask" ((d). Last, "Vascular centering map 2" $(\boldsymbol{m})$ is generated from the "Shaved recentered mask" $(\boldsymbol{g})$ by both dilation and Euclidean distance transformation.

Hair removal. The ill-conditioned nature of morphological thinning allows noisy boundaries of the vessel surface to be transformed into short free-ends, or hairs, on the center-line mask (Fig. $6 e, f$ ). Each center-line voxel with $>2$ neighbors is either the junction of two vessels or the attachment point of a hair. The removal of potential hairs is based on the length of the free-end relative to the radius of the vessel to which it is attached. This radius is found from the approximate radius map (Fig. 6j). We define a hair as a segment whose length is less than the radius of the associated vessel plus two voxels, and remove it. This process is iterated 

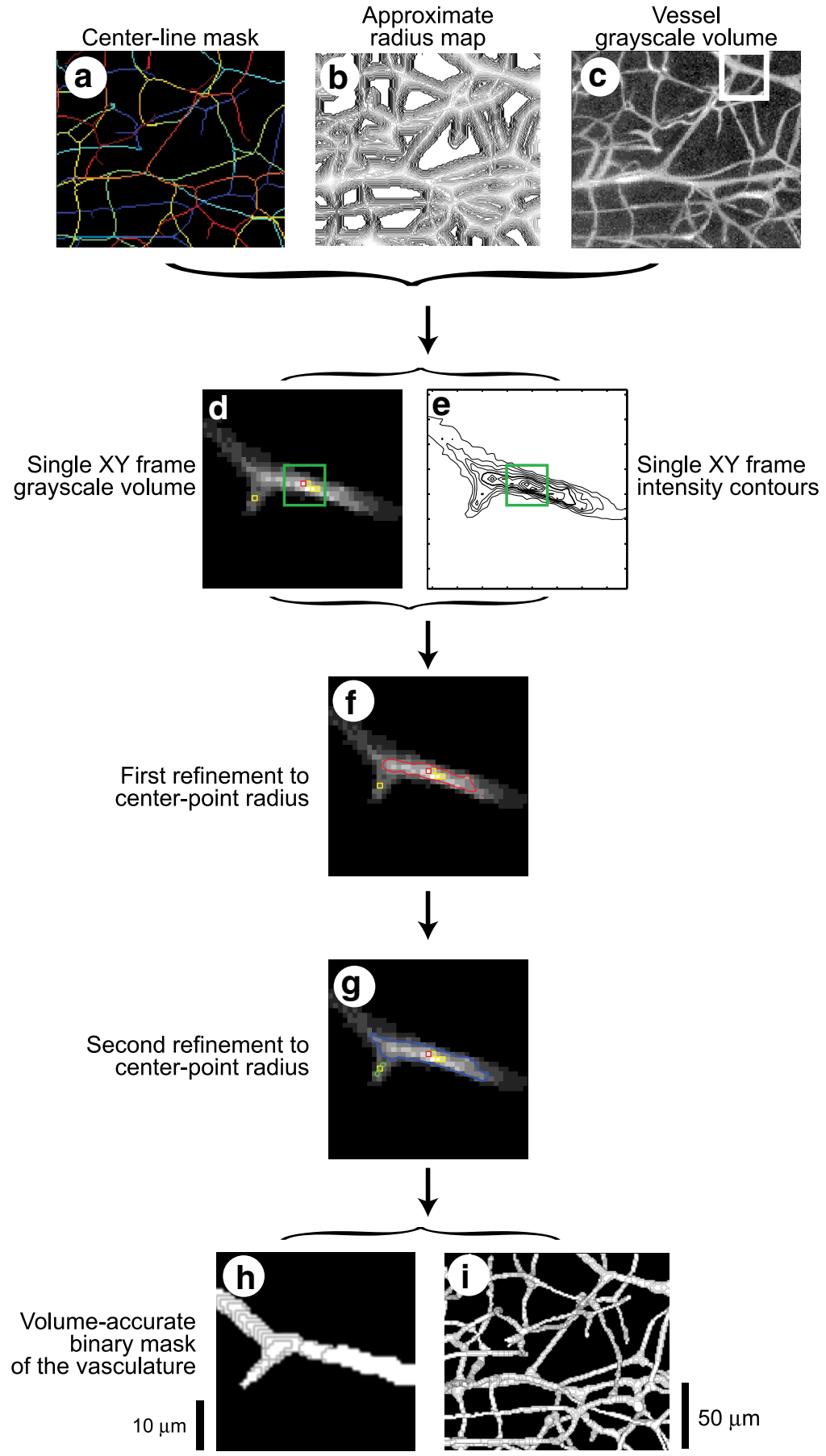

Figure 7. Process flow diagram for determination of vascular radii. $\boldsymbol{a}-\boldsymbol{c}$, Radii determination for each sub-block is performed using the appropriate "Center-line mask" (a) along with the corresponding "Approximate radius map" (b) and "Vessel grayscale volume" (c) generated in the vascular segmentation process (Fig. 6). The white box denotes the region expanded for panels (d) to (h). $\boldsymbol{d}, \boldsymbol{e}$, Gray-scale image $(\boldsymbol{d})$ and contour lines $(\boldsymbol{e})$ of the intensity in a single xy frame of the "Vessel grayscale volume" around center-line point (red square). The green box defines a local neighborhood around the center-line point. $f$, The first refinement to the estimate of the radius (red contour) calculated from the maximum of an Euclidean distance transform, as found from a threshold at a fixed percentage of the maximum intensity in the local neighborhood. $\boldsymbol{g}$, The second refinement to the estimate of the radius based on cylindrical vessels and an estimated point-spread function for the microscope. $\boldsymbol{h}, \boldsymbol{i}, \mathrm{A}$ volume-accurate vasculature binary mask is formed from overlapping spheres of the appropriate radii at each centerline point.

until no more hairs are present and results in the shaved recentered mask (Fig. $6 g$ ). Endpoints that fall outside the cutoff distance are putatively associated with true vessel segments and preserved.

Vascular centering map 2. This map is used to remove loops from the shaved recentered mask (Fig. $6 g$ ). The shaved and enhanced mask is dilated with five iterations of a $3 \times 3 \times 3$ voxel block. We then take a Euclidean distance transform of the dilated mask (Fig. $6 \mathrm{~m}$ ).
Small loop removal. Pixel-to-pixel noise in the grayscale data that propagate to the enhanced mask (Fig. $6 d$ ) can produce local variations in vasculature centering map 1 (Fig. 6l). This can lead to splitting of vascular centerlines so that short sections of a single vessel are split into parallel vessels and form a loop that would not be detected by the previous recentering process. We thus perform a second round of recentering using vasculature centering map 2 (Fig. $6 \mathrm{~m}$ ). This yields a final center-line mask (Fig. 6h).

\section{Vessel radii}

Vessels are assumed to have cylindrical symmetry with radii estimated from $x y$ planar images, which have less aberration than the $x z$ and $y z$ sections. A first estimate of radii for each center-line voxel along the vasculature (Figs. 6h,7a) is calculated from the approximate radius map (Figs. 6j, 7b). A twodimensional square neighborhood in the $\mathrm{XY}$ plane of the grayscale volume data (Figs. $6 a$, $7 c$ ), chosen as $18 \times$ the estimated radius of the vessel on edge, forms a region of interest around a point on the center-line (Fig. $7 c$, white box, $d, e)$. A smaller box, chosen as the larger of 9 pixels or $3 \times$ the radius on edge, is used to determine the peak intensity of the vessel (Fig. $7 d$,e, green box). The voxels in the larger square are thresholded at $0.4 \times$ the peak intensity to form a binary image that is morphologically closed with a $3 \times 3$ square kernel and upsampled by a factor of two. The maximum value of a Euclidean distance transform in the vicinity of the centerline pixel is taken as the second refinement of the radius for each center-line point (Fig. $7 f$, red contour).

The radius is further refined to account for the width of the PSF. For vessels whose diameters are much larger than the point spread function of the imaging system, the excitation volume will be half-in and half-out of the vessel when focused at a vessel edge. For vessels whose diameters approach the size of the PSF, a look-up table was constructed to define thresholds based on simulations with uniformly labeled cylindrical rods and an expanded PSF that approximated the broadened beam during deep imaging. The look-up table provides the fraction of the peak intensity that is appropriate to threshold vessels of different diameters. These thresholds range from $0.28 \times$ the maximum value to a limit of $0.50 \times$ the maximum that is reached when the width of the vessel is large compared to that of the PSF, i.e., $0.6 \mu \mathrm{m}$ along the $x$-and $y$-axes and $4 \mu \mathrm{m}$ along the $z$-axes at depths of $500 \mu \mathrm{m}$. The thresholded image is morphologically closed with a $3 \times 3$ square kernel, supersampled by a factor of two, and the maximum value of a distance transform in the vicinity of the centerline point is taken as the second and final estimate of radius (Fig. $7 g$ ). A volume-accurate binary mask of the vasculature is reconstructed by the overlapping placement of spheres of the appropriate radii at each centerline point (Fig. $7 h, i)$.

\section{Neuron classification}

The VIDA suite is used to classify each cell nucleus based on the on the colocalized signals in the DAPI and Alexa 594 channels (Fig. 3f,h).

Secondary spectral demixing 2. A second round of refinement to the demixing of data channels makes use of the results of the vasculature and 
nuclear segmentation. First, the raw vascular mask (Fig. 6i) is used to refine the spectral demixing coefficient for removal of fluorescent signals from vasculature that still contaminate the Alexa 594 data after the first round of demixing; this refined data forms the neuronal nuclei grayscale volume. Second, we form the ratio between the spatially filtered DAPI channel, using a $3 \times 3 \times 3$ voxel block, and the spatially filtered neuronal nuclei grayscale volume. The top $0.1 \%$ of voxels with the largest value of this ratio form a set of clusters that highlight some non-neuronal nuclei and define a ratio mask. Next, we define a background mask as a concentric shell outside each object in the ratio mask; the inner radius of the shell is found by dilating the binary cell mask (Fig. $5 c$ ) by one voxel and the outer radius is found by dilating the ratio mask. We next mask the Alexa 594 grayscale volume intensities with the ratio mask and compute the median value for each object. Similarly, we apply the background mask to the Alexa 594 grayscale volume and compute the median value of these voxels. The Alexa 594 grayscale volume is the further demixed to minimize the difference the above median values.

Sorting based on relative staining. Each cell nucleus in the cell mask from the DAPI channel (Fig. 5) was evaluated individually. The mean intensity, denoted $I_{\text {cell }}$, of the corresponding voxels in the neuronal nuclei grayscale volume was calculated, as well as the mean background intensity, $I_{\mathrm{bkg}}$, in a spherical shell that surrounds the evaluated cell. The shell is formed by dilating the value of the mask by 2 voxels and is 2 voxels thick; dilated masks of the other nearby cell nuclei and vasculature are excluded from the shell to avoid contamination of the background reading (Fig. 8a). A local neuronal signal value is calculated as $q_{\mathrm{i}}^{(\alpha)} \equiv I_{\text {cell }}-I_{\text {bkg }}$, where $\alpha$ designates the nucleus as neuronal, $\alpha=\mathrm{n}$, or non-neuronal, $\alpha=$ nn (Fig. $8 b$ ). Cells that are not labeled above background staining levels result in signal values close to zero. Cells that are labeled above background staining levels result in positive signal values whose magnitude depends on staining and imaging conditions.

To compensate for large low spatial frequency variations in the staining intensity across the tissue, an iterative, locally ratiometric analysis is performed to evaluate $q_{\mathrm{i}}^{(\alpha)}$ relative to the same metric for all neighboring nuclei in a $80 \times 80 \times 80$ voxel region that is centered on the nucleus of interest. Initially all nuclei are classified as neuronal nuclei. We then consider the ratio between the value of $q_{i}^{(\alpha)}$ for the cell under evaluation compared to neighboring cells that are currently classified as neurons, i.e., $q_{\mathrm{i}}^{(\alpha)} /\langle q(n)\rangle$, where

$$
\left\langle q^{(\mathrm{n})}\right\rangle \equiv \frac{1}{N_{\text {neurons }}} \sum_{j=1}^{N_{\text {neurons }}} q_{j}^{(\mathrm{n})},
$$

and $N_{\text {neurons }}$ is the current estimate of the number of neuronal nuclei in the neighborhood. Nonlabeled cells result in values for the ratio that are close to zero, while labeled cells result in values close to unity. The resulting histogram for the ratio is fit to a mixture of two Gaussians, and a threshold ratio value is determined by maximum likelihood estimation. Each cell is then reclassified as neuronal or non-neuronal based on this threshold ratio. This ratiometric reclassification is repeated until $<0.2 \%$ of nuclei are reclassified between rounds (Fig. 8 c).
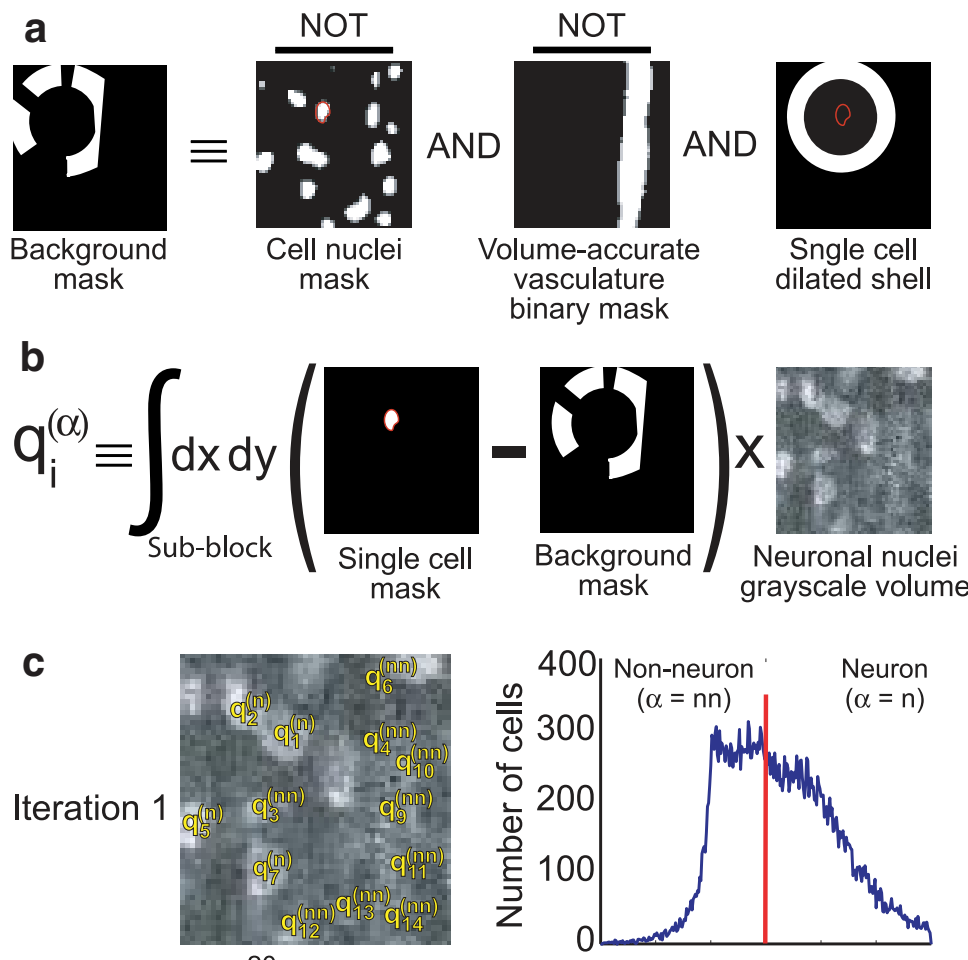

$20 \mu \mathrm{m}$
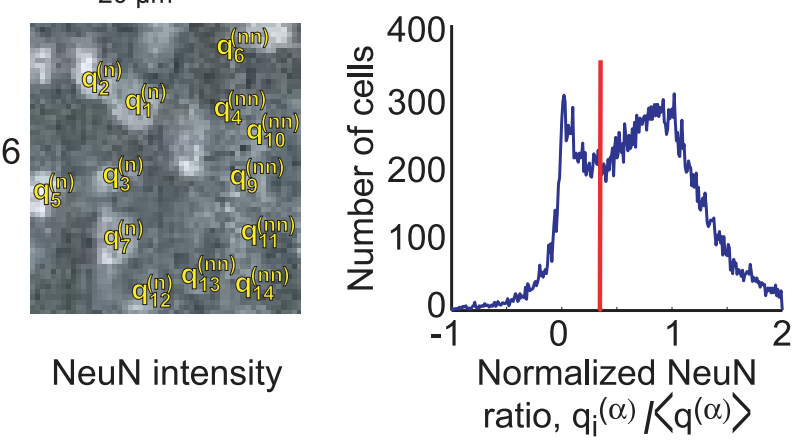

NeuN intensity

$$
\text { ratio, } q_{i}{ }^{(\alpha)}\left\langle q^{(\alpha)}\right\rangle
$$

Figure 8. Process flow for discrimination of neuronal versus non-neuronal cell nuclei. $\boldsymbol{a}$, A background mask is generated for each cell nucleus. A dilated shell of the target cell's nucleus mask is intersected with the inverse of the full "Cell nucleus mask" (Fig. $5 c$ ) and the inverse ucleus and " $\alpha$ " signifies neuronal or non-neuronal. $\boldsymbol{c}$, An iterative process is applied to classify each nucleus as neuronal or non-neuronal based on the ratio of the value of $q_{i}^{(\alpha)}$ relative to the mean value of its local neuronal neighbors. The classification of each nucleus is subject to change at each iteration, and only those nuclei currently classified as neurons contribute to the mean value.

\section{Microvessel classification}

A separate binary mask of microvessels is made from the mask of all vessels in which only those vessel regions whose local diameter is $<6 \mu \mathrm{m}$ are kept. This value was observed to be the inflection point in a histogram of the radii of all vessels.

\section{Montaging}

All sub-block masks are restitched to form montaged masks. The assembly of sub-blocks is based on the coordinates of the original sub-blocks. In the overlapped regions, we compute the midpoint of the overlap and keep those voxels that belong to the nearest sub-block. Cell nuclei coordinates and classifications are compiled together into a master list. Centroids that were spaced closer than $D_{\text {cell_min }}$, were removed using the same algorithm used for centroid elimination within a sub-block, as discussed above.

\section{Analysis}

Cell number densities were computed by summing the number of nuclei across the full width and thickness of the slab in a $50 \mu \mathrm{m}$ wide sliding window in the radial direction and dividing by the volume of tissue. This 
a

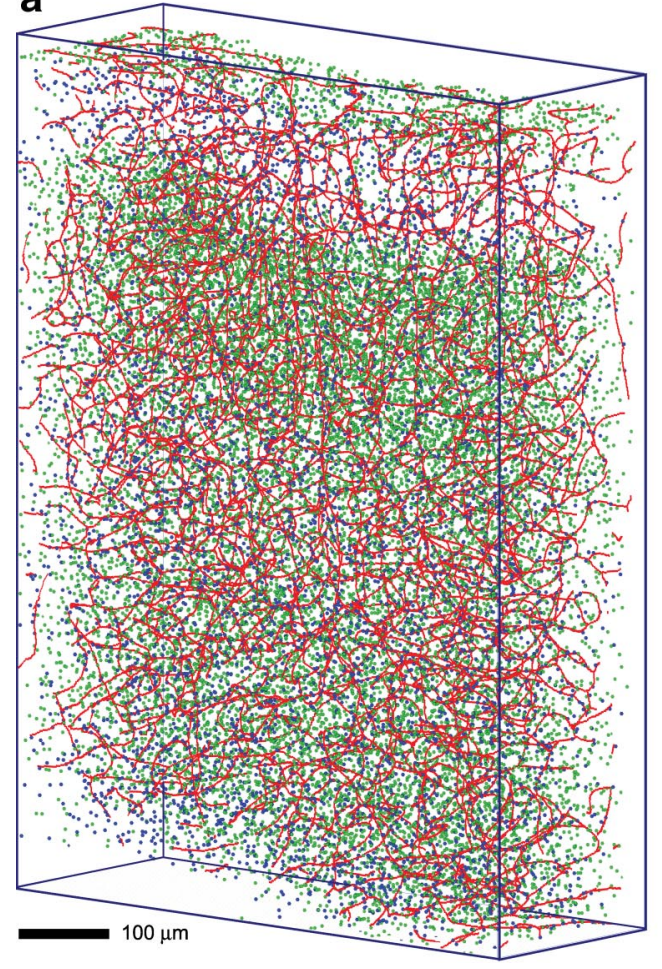

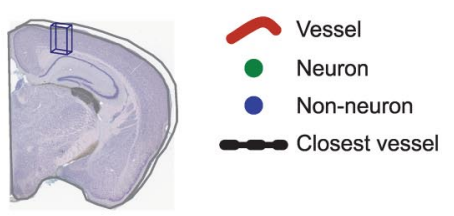

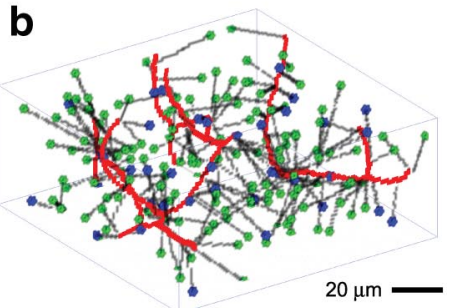

C

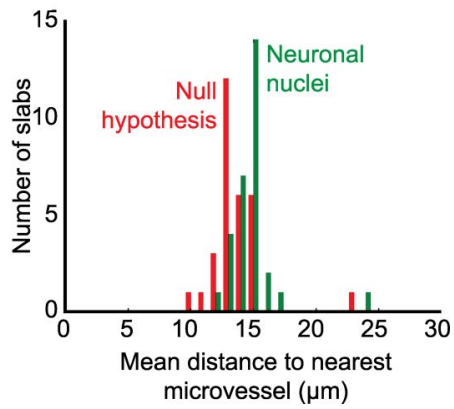

Figure 9. Volumetric reconstruction of vasculature and cell nuclei. A $900 \times 650 \times 250 \mu \mathrm{m}^{3}$ volume of cortical tissue from a sagittal section. The volume is centered at $2 \mathrm{~mm}$ caudal of bregma and $1.1 \mathrm{~mm}$ lateral of the midline. $\boldsymbol{a}$, The montaged centerline mask (red) overlaid with $3 \mu \mathrm{m}$ spheres at the centroid location for each neuronal (green) and non-neuronal (blue) nucleus. The side figure illustrates the orientation of the volume. $\boldsymbol{b}$, Expanded view of sub-region of $\boldsymbol{a}$. Black lines indicate the nearest vascular component to each cellular nuclei. For clarity, only vascular components within the visualized sub-region were considered; cellular nuclei near the sub-region border may be closer to a vascular component that is out of the current view. c, Histogram of the distances between all neuronal nuclei in a slab to the nearest microvascular component (green). The null hypothesis is the distribution of all distances beyond a minimum possible distance to the nearest vessel (red). The two curves are statistically different.

yields the number of nuclei per $\mathrm{mm}^{3}$ as a function of radial depth into cortex. Areal densities were computed by multiplying the number density by the radial depth of the slab; this normalizes against variations in the thickness of the cortical mantel.

Fractional volume density of vasculature was similarly computed by summing the volume of the blood vessels across the full width and thickness of the slab in a $50 \mu \mathrm{m}$ wide sliding window in the radial direction and dividing by the volume of tissue. This yields the fraction of the cortex occupied by the lumen of the vessel as a function of radial depth into cortex. Normalized vascular length was computed by summing the length of the centerlines of blood vessels in a $50 \mu \mathrm{m}$ band across the fill width and thickness of the slab; the center-to-center distance is $1.00 \mu \mathrm{m}$ for voxels that touch face-to-face, $1.41 \mu \mathrm{m}$ for voxels that touch edge-toedge, and $1.73 \mu \mathrm{m}$ for voxels that touch corner-to-corner. This yields the length of vasculature per $\mathrm{mm}^{3}$ of cortex as a function width radial depth into cortex.

\section{Results}

Reconstructed architectonics of all neurons and non-neurons was obtained in register with angioarchitecture from millimeterthick slabs of neocortex (Fig. 3). The location of each nucleus is defined by a vector and the centerline of each vessel is defined by a set of vectors, where each component is additionally tagged with size and type information (Fig. 9). The vectorization of all nuclei and vessels allowed us to test for correlations in their densities. The first dataset was used to examine the relationship between cells and vessels as a function of the radial depth from pia to white matter, as well as provide total densities for comparisons between animals (Fig. 10). Our samples include one volume from frontal cortex and three volumes from parietal cortex, one of which is from the vibrissa ("barrel") area. These locations contain different levels of granularity, a qualitative measure that is discerned from medium- and high-resolution images of the areas of interest (supplemental Fig. 2, available at www.jneurosci.org as supplemental material). The second set was used to examine the relationship between cells and vessels as a function of cortical area and consisted of coronal slabs that sampled parietal and insular cortex (Fig. 11).

Nuclear stains are used to identify all cell bodies, and labeled antibodies to the perinuclear protein $\mathrm{NeuN}$ are used to segregate neurons and non-neurons (see Materials and Methods). Mechanically stable fluorescent gels are used to fill the vasculature and a break in size at a diameter of $6 \mu \mathrm{m}$ is used to demarcate vessels as microvessels (see Materials and Methods). All labels were optimized to stain the entire thickness of the slab (supplemental Fig. 1, available at www.jneurosci.org as supplemental material), which was imaged with TPLSM (Fig. 3). A suite of rigorously tested numerical routines that used matched filtering and morphometric tools was used to automatically locate and count every cell nuclei and to concurrently map the backbone and radii of all vessels (Figs. 4-9).

\section{Spatial distributions of cells and vessels}

The locations of all nuclei (Fig. 5), their assignment as a neuron (Fig. 8), and the center-line and diameter of all vessels (Figs. 6, 7) were calculated for every slab. The results for a portion of a particular slab in parietal cortex are shown in the reconstruction of Figure $9 a$. We consider first the statistics as an average over all layers and slabs, i.e., number density of neuronal nuclei $=$ $(1.17 \pm 0.27) \times 10^{5}$ nuclei per $\mathrm{mm}^{3}$; number density of nonneuronal nuclei $=(0.43 \pm 0.26) \times 10^{5}$ nuclei per $\mathrm{mm}^{3}$; areal density of neuronal nuclei $=(1.41 \pm 0.34) \times 10^{4}$ nuclei per $\mathrm{mm}^{2}$; fractional vascular volume $=0.0097 \pm 0.0021 \mathrm{~mm}^{3}$ per $\mathrm{mm}^{3}$; normalized vascular length $=0.88 \pm 0.17 \mathrm{~m}$ per $\mathrm{mm}^{3}$; fractional microvascular volume $=0.0074 \pm 0.0014 \mathrm{~mm}^{3}$ per $\mathrm{mm}^{3}$, where we report the mean and SD.

An expanded view of the reconstructed images shows that the distance for each cell body to the nearest blood vessel is readily calculated (Fig. 9b, dashed lines). Across all sagittal slabs (36 slabs in 12 animals), we find that the distance from the neuronal nuclei to the nearest microvessel is $14.6 \pm 1.0 \mu \mathrm{m}$ (mean \pm SD) (Fig. $9 c$ ). By comparison, the null hypothesis that the location of cells in unaffected by that of microvessels yields a distance from the neuronal nuclei to the nearest microvessel of $13.3 \pm 1.2 \mu \mathrm{m}$; in both cases we exclude all points closer than a minimal closest approach of one microvessel plus one cell soma radius, i.e., $5 \mu \mathrm{m}$ from the centerlines. These data support a tendency for neuronal nuclei to 
lie further away from vessels than would be expected from a random spatial distribution (Fig. 9c). The effect is small, i.e., 9\%, yet statistically significant $\left(p<10^{-2}\right)$.

\section{Neuronal-vascular covariation}

The number density of all cell nuclei (50 slabs in 18 animals) and neuronal nuclei (41 slabs in 15 animals), and the fractional volume density of all vasculature ( 50 slabs in 18 animals) were calculated as a function of radial depth for all slabs. We arrange the data for the different regions as a progression from agranular to granular regions (Fig. 10a-h), which nominally corresponds to a progression of increasing metabolic load. The number density for neuronal nuclei demonstrates the expected variation across cortical layers, with an increase in the density at the expected location of layers 4 and 6 (Fig. $10 a-d$, green band); the effect is particularly evident in granular cortex (Fig. 10d), a primary sensory area. In contrast, the non-neuronal nuclei, which include both glial and endothelial cells, remains relatively constant across the cortical layers (Fig. $10 a-d$, blue band) at all locations.

We turn now to the cortical vasculature (Fig. 10e-h). The total vascular volume exhibits a peak at the cortical surface as a result of the presence of large surface vessels then rapidly decreases within the first $100 \mu \mathrm{m}$ below the pia, followed by a more gradual decrease with increasing depth into cortex. In contrast, the microvascular volume density varies relatively little across cortex, with a shallow maximum near $600 \mu \mathrm{m}$ below the pial surface. On average, this corresponds to a gradual increase of $\sim 15 \%$ from layer $2 / 3$ to layer 5 before dropping by $\sim 7 \%$ at layer 6 . Critically, there is no tendency to match the distribution of neuronal nuclei (Fig. 10, compare $e-h, a-d$ ), even in the vibrissa area (Fig. 10, compare $h, d$ ).

While the above data show that nuclei and vessels do not track throughout the depth of cortex, we tested if the summed densities covaried on each of two global scales. First, in terms of average densities across animals and slabs $(n=45)$, we find a positive and statistically significant correlation $\left(r=0.64, p<10^{-5}\right)$ between the fractional volume of the vasculature and the areal density of neuronal nuclei (Fig. 10i). The related correlation between the normalized vascular length and the areal density of neuronal nuclei is even stronger $\left(r=0.75, p<10^{-7}\right)$ (Fig. $10 j)$. These correlations are also seen, more weakly but significantly, when the number density rather than the areal density is considered.

Second, we checked the hypothesis that the average neuronal number density and vasculature volume density, when averaged over the full thickness of cortex, covary across different areas of cortex within the same animal. Here, we used coronal sections from perfused and labeled animals at a level just caudal to bregma (Fig. 11a). For purposes of analysis, we arrayed the data as neighsignificantly correlated.

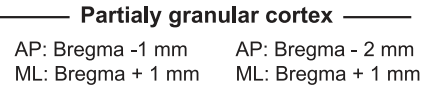

Granular cortex AP: Bregma - $1 \mathrm{~mm}$ $\mathrm{ML}:$ Bregma $+4 \mathrm{~mm}$

b

C
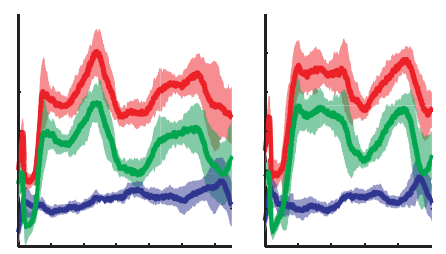

d

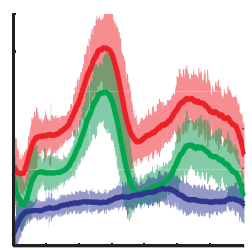

g

h
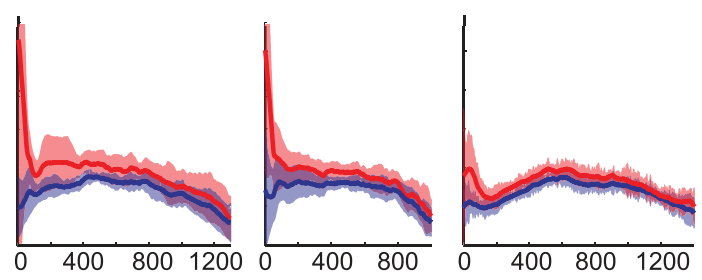

Depth below cortical surface $(\mu \mathrm{m})$
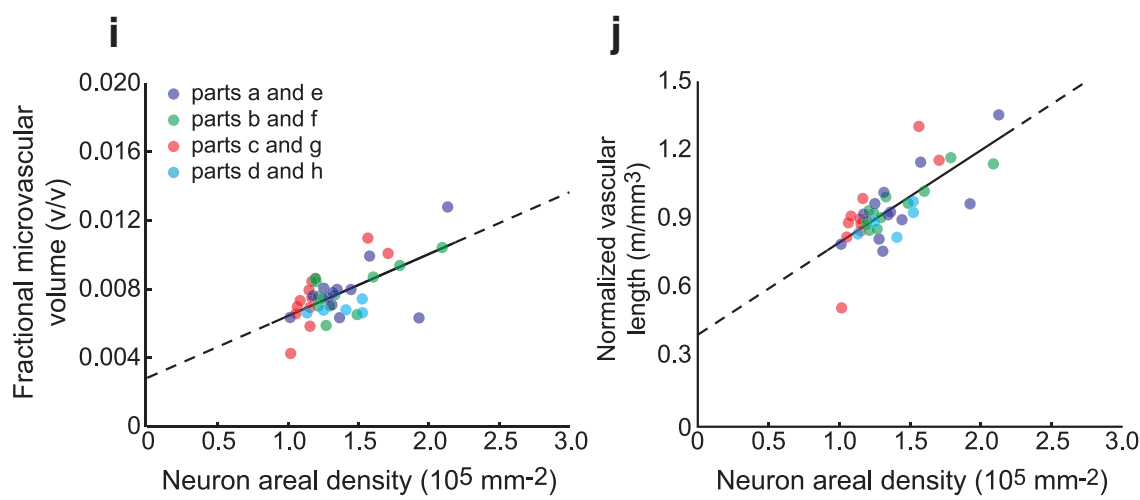

Figure 10. Vascular and cellular density plots from thick sagittal sections. Vascular and cellular densities are computed for the processed data from thick sagittal sections with center coordinates as noted. $\boldsymbol{a}-\boldsymbol{d}$, The number densities of all cells (red), neurons (green), and non-neurons (blue) per $\mathrm{mm}^{3}$ of tissue as a function of cortical depth. $\boldsymbol{e}-\boldsymbol{h}$, The fractional volume occupied by all

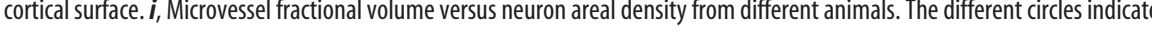
from locations in $\boldsymbol{a}-\boldsymbol{d}$ as noted. The microvascular density and neuron areal density are significantly correlated.

boring wedges and segmented the vasculature, neuronal and non-neuronal cells in each wedge. We observe a trend for both neuronal and microvascular densities to decrease as one moves laterally along the cortical mantle from cingulate to entorhinal cortex (Fig. 11b,c). Critically, both the volume fraction of the microvasculature and the length of the vasculature increase in step with the neuronal number density $\left(r=0.63, p<10^{-52}\right.$ and $r=0.68, p<10^{-62}$, respectively) (Fig. $11 d, e$ ).

\section{Controls on metrology}

We performed a number of tests to estimate systematic errors in both our measurements and our data analysis.

\section{Comparison with manual counts}

We manually tagged the nuclei in a $200 \times 200 \times 200 \mu \mathrm{m}^{3}$ volume of the DAPI channel using a three-dimensional display. For simplicity, we avoided all nuclei that were bisected at a boundary. Our automatic segmentation scheme (Fig. 6) recalled $97 \%$ of the $\sim 1800$ nuclei that we identified manually, with a precision of $95 \%$. 


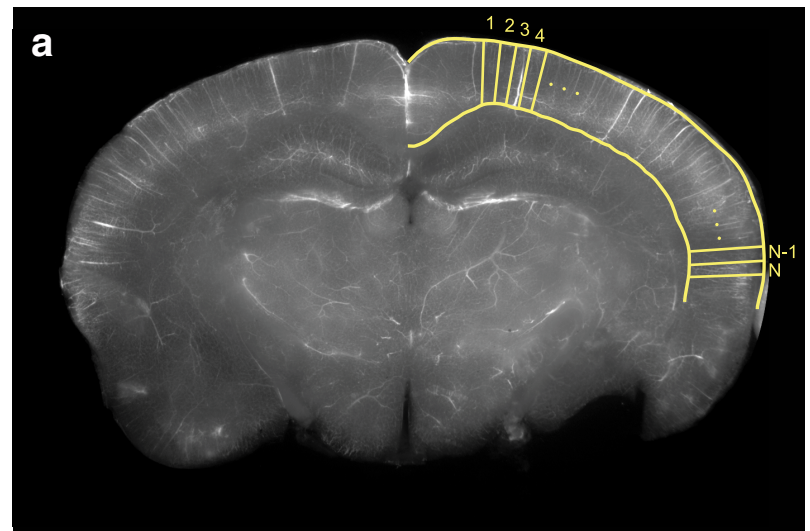

b

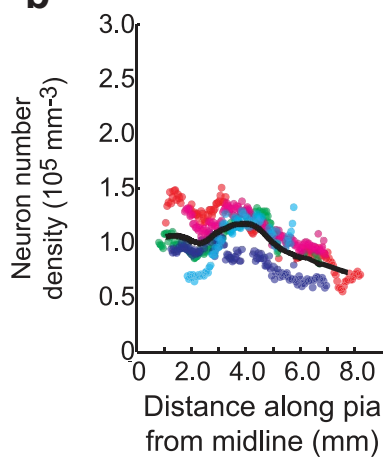

C

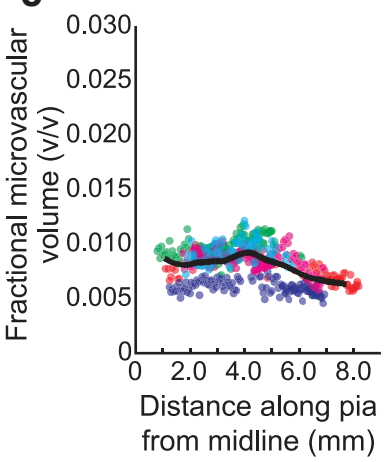

d

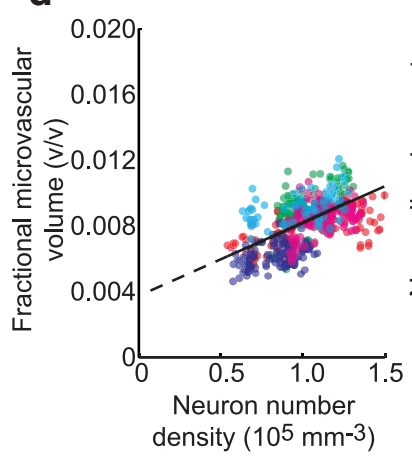

e

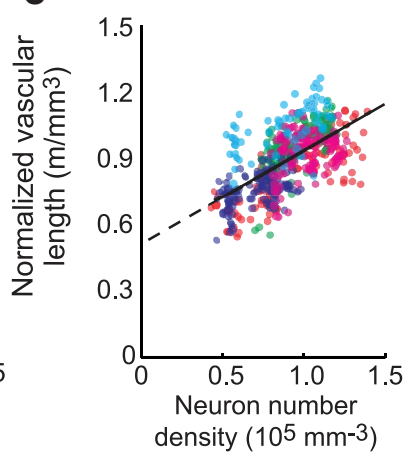

Figure 11. Vascular and cellular density plots from thick coronal sections. Vascular and cellular densities are computed for the processed data from thick coronal sections. $\boldsymbol{a}$, A microphotograph of a $1 \mathrm{~mm}$ thick coronal section between 0 and $-1 \mathrm{~mm}$ relative to bregma in which the vasculature has been labeled by fluorescent gel perfusion. After staining and clearing, the entire cortex is imaged and processed as individual wedges, $w_{1}$ through $w_{N}$, that run perpendicular to the pial surface and span the depth of cortex. The spacing and width of the wedges is $50 \mu \mathrm{m}$ along the pial surface. $\boldsymbol{b}$, Neuronal number density versus distance from the midline along the pial surface. $\boldsymbol{c}$, Fractional microvascular volume plotted versus distance from the midline. Each dot in $\boldsymbol{b}$ and $\boldsymbol{c}$ represents the mean density across a single wedge. Different colored dots represent different coronal sections ( $n=5$ sections across 2 animals). The black lines are a 50-point running average across all coronal sections. $\boldsymbol{d}$, Fractional microvascular volume plotted versus neuronal number density. $\boldsymbol{e}$, Normalized vascular length plotted versus neuronal number density. The black lines in $\boldsymbol{d}$ and $\boldsymbol{e}$ represent a linear regression across all points.

In vivo confirmation of microvessel diameter

To verify that the vessel diameters we report for our histological reconstructions are physiologically accurate, we analyzed image stacks of labeled vasculature obtained through a thinned-skull preparation of an awake mouse. We find the distribution of the microvessels diameters of the histological preparations to be comparable with that seen in vivo (supplemental Fig. 3, available at www.jneurosci.org as supplemental material). The average di- ameters of in vivo and histological microvessels are $3.97 \mu \mathrm{m}$ and $4.11 \mu \mathrm{m}$, respectively.

\section{Sensitivity analysis}

Our analysis algorithms contain 19 parameters for the segmentation and categorization of nuclei and 41 parameters for the segmentation, center-lining, and volumetric determination of blood vessels (supplemental Tables 1-5, available at www.jneurosci.org as supplemental material). Their values were chosen based on the results of a number of quantitative tests to place the operating points for the parameters in a relatively insensitive range and, for the case of nuclei, to conform with "ground truth" estimates of cell numbers. We checked the sensitivity of our results to changes in the parameters by systemically changing the value of each parameter by $\pm 20 \%$ and $\pm 50 \%$ and recalculating the results of our analysis.

For total nuclear counts and counts of only neuronal nuclei, we report the fractional change in number, denoted $\delta n / n_{\mathrm{o}}$, where $n_{\mathrm{o}}$ is the baseline estimate of the number per unit volume, with respect to the fractional change in a parameter, denoted $\delta p / p_{0}$ where $p_{\mathrm{o}}$ is the baseline value of the parameter. These fractions are related by the following:

$$
\frac{\delta n}{n_{0}} \cong \frac{\partial\left(n / n_{0}\right)}{\partial\left(p / p_{0}\right)} \frac{\delta p}{p_{0}}+\frac{1}{2} \frac{\partial^{2}\left(n / n_{0}\right)}{\partial\left(p / p_{0}\right)^{2}}\left(\frac{\delta p}{p_{0}}\right)^{2},
$$

where the derivatives are found empirically and reported in supplemental Tables 1, 2, available at www.jneurosci.org as supplemental material. From the perspective of sensitivity to small changes in a parameter, the number density is most sensitive to the radius of the matched filter (Fig. $5, \sigma_{\text {cell-local }}$ ), for which a $10 \%$ change in the parameter $\left(\delta \mathrm{p} / p_{\mathrm{o}}=0.10\right)$ leads to a $1.5 \%$ change in number density $\left(\delta n / n_{\mathrm{o}}=0.015\right)$. From the perspective of sensitivity to small changes in a parameter, the linear error term in Equation 2 dominates and the most sensitive parameters for the matched filter have a 1:8 to 1:10 ratio. A similar argument holds for the choice of $D_{\text {cell_min }}$, the threshold distance to decide if two centroids are merged, $D_{\text {cell_min }}$, a 1:10 effect (supplemental Table 1, available at www.jneurosci.org as supplemental material; Fig. $5 d, e)$. Changes in all other parameters had 1:20 to 1:100 effects on the number density. Further, changes in the parameters associated with the classification of a nucleus as a non-neuron or neuron had a negligible effect, i.e., <1:50 (supplemental Table 2, available at www.jneurosci.org as supplemental material). All told, our estimate of the number density appeared to be dominated by volume-to-volume variability rather than systematic errors (Fig. 10a-d).

For estimates of vascular volume, we report the fractional change in volume, denoted $\delta v / v_{\mathrm{o}}$ where $v_{\mathrm{o}}$ is the baseline estimate of the volume, with respect to the fractional change in a parameter. These fractions are related by the same equation for the number density (Eq. 2) but with $n / n_{\mathrm{o}}$ replaced by $v / v_{\mathrm{o}}$; the derivatives are found empirically and reported in supplemental Tables 3-5, available at www.jneurosci.org as supplemental material. We find that the estimate of the backbone of the vasculature had a negligible effect, i.e., $<1: 50$, on the estimate of the microvascular volume (supplemental Table 3, available at www.jneurosci. org as supplemental material). The largest contribution occurs for the size of range used to adjust the centerline, so that a onehalf voxel shift leads to $\delta p / p_{\mathrm{o}}=0.17$, for which to $\delta v / v_{\mathrm{o}}=0.03$ (supplemental Table 4, available at www.jneurosci.org as supplemental material). From the perspective of sensitivity to small changes in a parameter, so that the linear error term dominates, 

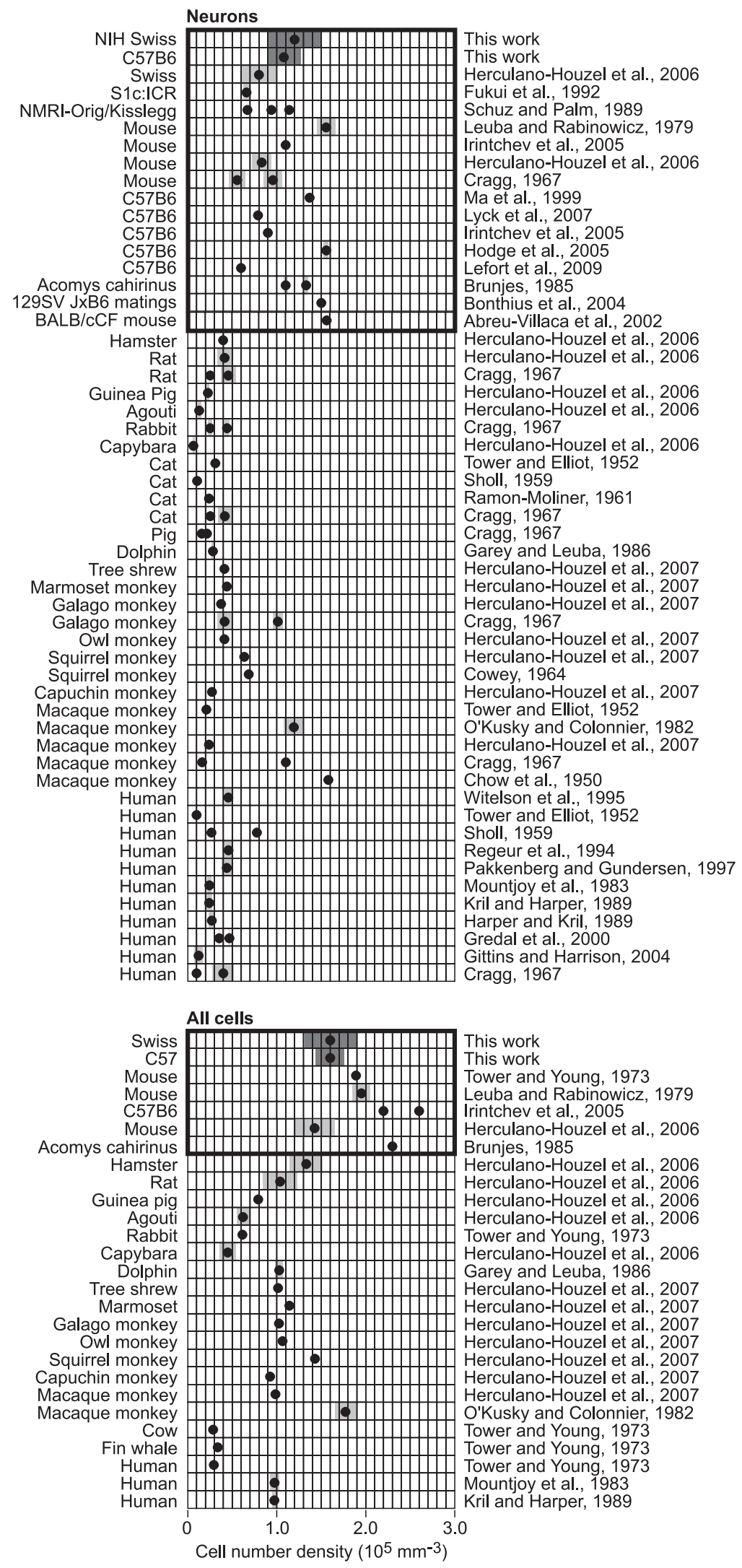

Figure 12. Compendium of literature results on neuronal and all cell densities. Data for mouse are derived from the present and published work (Leuba and Rabinowicz, 1979; Brunjes, 1985; Schüz and Palm, 1989; Fukui et al. 1992, Ma et al., 1999; AbreuVillaça et al., 2002; Bonthius et al., 2004; Hodge et al., 2005; Irintchev et al., 2005; Herculano-Houzel et al., 2006; Lefort et al., 2009; Lyck et al., 2007). Data for other species are derived from the original published work (Cragg, 1967; Tower and Young, 1973; 0'Kusky and Colonnier, 1982; Mountjoy et al., 1983; Barey and Leuba, 1986; Garey and Leuba, 1986; Harper and Kril, 1989; Kril and Harper, 1989; Regeur et al., 1994; Witelson et al., 1995; Pakkenberg and Gundersen, 1997; Gredal et al., 2000; Gittins and Harrison, the most sensitive parameters for the analysis of the radial contribution have 1:6 to 1:10 ratios (supplemental Table 4 , available at www.jneurosci.org as supplemental material). Furthermore, changes in the parameters associated with the classification of a vessel as a microvessel had a negligible effect, i.e., <1:50 (supplemental Table 5, available at www.jneurosci.org as supplemental material). All told, our estimate of the microvascular volume was dominated by a composite 20\% systematic error rather than volume-to-volume variability (Fig. 10e-h).

\section{Discussion}

We used a set of established and novel techniques to fluorescently label the vasculature, neuronal nuclei, and non-neuronal nuclei in millimeter-thick slabs of tissue (Figs. 1, $3)$. Volumetric optical sections of the fluorescent markers throughout the depth of the slab were obtained with two-photon laser scanning microscopy (Fig. 3), from which we construct profiles of cell and vascular density and, critically, quantify the covariation of neuronal and vascular density over relatively large areas of tissue without the need to sample only selected parts of the tissue (Figs. 10,11).

We find that the average distance of any location in cortex to a nearby microvessel is $13 \mu \mathrm{m}$ (Fig. 9), and that there is a tendency for neurons to be $9 \%$ further away from vessels than chance (Fig. 9c). This small difference is not inconsistent with a bias for non-neurons, such as astrocytes, to be closer to vessels. A critical issue concerns the flow of oxygen and nutriments. Zhang and Murphy (2007) found that dendrites remain viable if they are within $80 \mu \mathrm{m}$ of a microvessel. Thus, there is a considerable safety factor between the normal density of microvessels and a reduced density that will lead to hypoxia and neuronal degeneration.

While neurons have a tendency to cluster in layer 4, a major target of thalamic input, the layer-to-layer variations in neuronal density as a function of cortical depth is not sharply mirrored by either the non-neuronal density (blue trace, Fig. $10 a-d$ ) or the microvascular density (Fig. $10 e-h$, blue trace). Rather, the microves-

$\leftarrow$

2004; Herculano-Houzel et al., 2006, 2007) or, for the cases of Chow et al. (1950), Tower and Elliot (1952), Sholl (1959), Ramon-Moliner (1961), and Cowey (1964) obtained from the compilation in the study by Cragg (1967). For all datasets, the black dots represent mean values. For data from this work, dark gray bars encompass the SD. For previously published data, light gray bars encompass the SD and the absence of bars implies that the SD is unknown. 

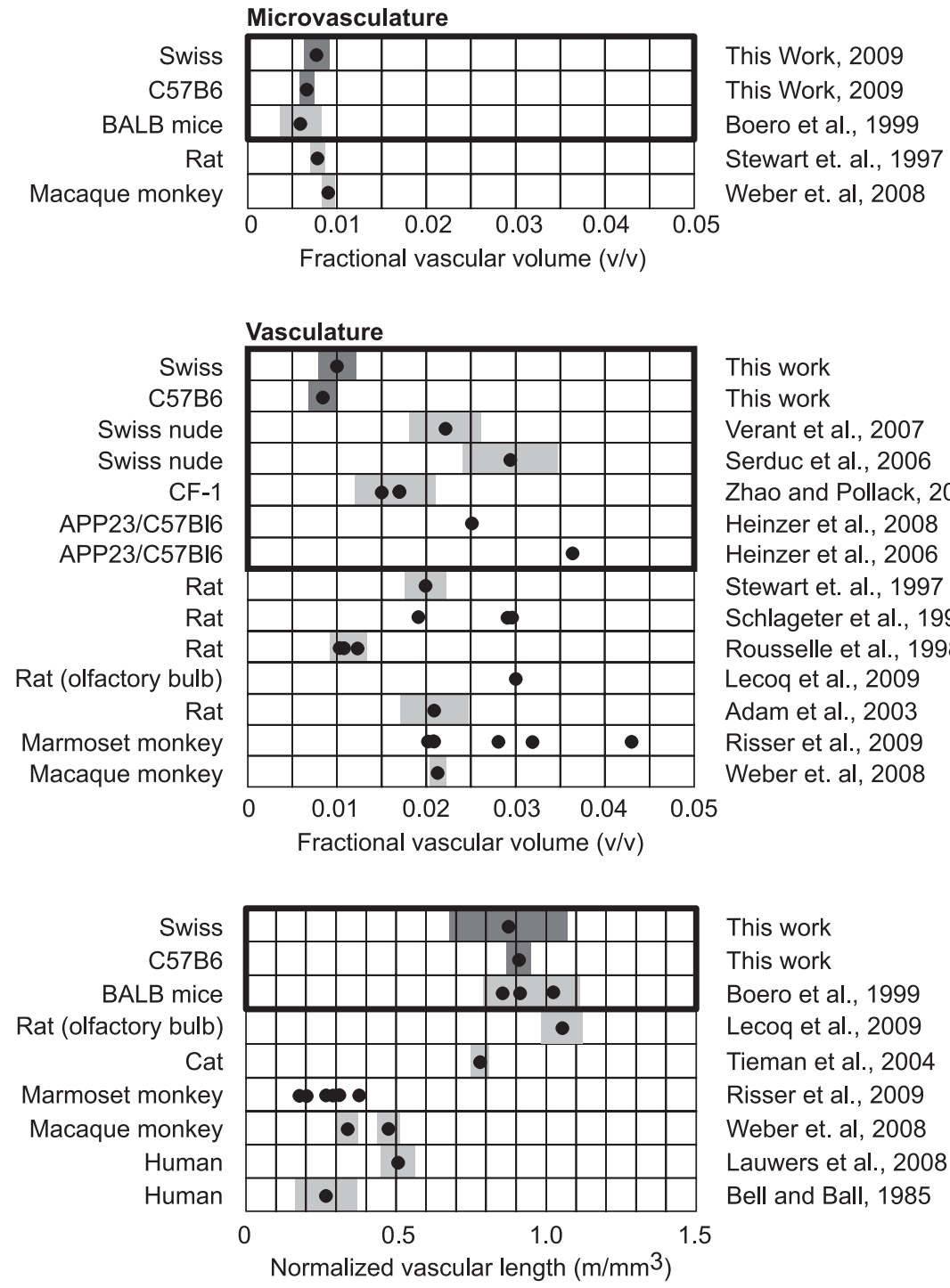

This work

This work

Boero et al., 1999

Lecoq et al., 2009

Tieman et al., 2004

Risser et al., 2009

Weber et. al, 2008

Lauwers et al., 2008

Bell and Ball, 1985

Normalized vascular length $\left(\mathrm{m} / \mathrm{mm}^{3}\right)$ there is apparently little increase in the carrying capacity of the vasculature to meet those needs in mice and possibly monkeys and man. This suggests that the red blood cells in layer 4 would be depleted of oxygen relatively quickly in response to a strong sensory stimulus, consistent with reports of a rapid, initial drop in blood oxygenation in the middle layer of cortex (Silva and Koretsky, 2002).

In contrast to the lack of close variation between neuronal and vascular densities on a laminar basis, we find that the density of neurons and blood vessels are positively correlated on a regional basis. This occurs within the same animal (Fig. 11d,e) and across animals (Fig. $10 i, j)$, so that regions with a high vascular count have high cell counts. The observation that vascular and cellular densities correlate across animals and brain regions, but not across cortical layers, suggests that gradients in signaling factors (Lok et al., 2007) that influence both vascular and cellular growth occur on millimeter scales but not submillimeter scales. This is consistent with the finding that blood vessels are patterned early in cortical development based on neuronal density in the subplate (Carmeliet and Tessier-Lavigne, 2005; Stubbs et al., 2009).

\section{Comparison with past cell counts}

We find the average density of all cells across cortical layers to be $1.6 \times 10^{5}$ cells per $\mathrm{mm}^{3}$ and falls within the range of values previously reported in literature, i.e., $1.5 \times 10^{5}$ (Herculano-Houzel et al., 2006) to $2.6 \times 10^{5}$ cells per $\mathrm{mm}^{3}$ (Irintchev et al., 2005) (Fig. 12). We find the average density of neurons across cortical layers to be $1.2 \times 10^{5}$ neurons per $\mathrm{mm}^{3}$, which also falls within the range of reported values, i.e., $0.6 \times 10^{5}$ to $1.6 \times 10^{5}$ neurons present and published work (Boero et al., 1999; Heinzer et al., 2006, 2008; Serduc et al., 2007; Vérant et al., 2007; Zhao and Pollack, 2009). Data for other species are derived from published work (Bell and Ball, 1985; Stewart et al., 1997; Rousselle et al., 1998; Schlageter et al., 1999; Tieman et al., 2004; Lauwers et al., 2008; Weber et al., 2008; Lecoq et al., 2009; Risser et al., 2009). For all datasets, the black dots represent mean values. For data from this work, dark gray bars encompass the SD. For previously published data, light gray bars encompass the SD and the absence of bars implies that the SD is unknown.

sels have a shallow rise that peaks near layer 4 and extends across the entire column. This peak is more prominent in granular regions of cortex (Fig. 10e), yet less pronounced than found in a previous analysis for rat cortex (Patel, 1983). Laminar variations in vascular density similar to that found here has been reported for monkey cortex (Weber et al., 2008; Risser et al., 2009), including tissue from primary visual cortex, and human cortex based on postmortem data (Cassot et al., 2006; Lauwers et al., 2008). Yet there is considerable discord among different reports with respect to the extent that the vasculature follows laminar variations in cell density, with an early report indicating a strong demarcation in monkey (Zheng et al., 1991), while other reports for monkey (Weber et al., 2008) and human (Bell and Ball, 1985) indicate only that the vascular density has a trough at the position of layer 5 .

Cortical layer 4 is expected to have the highest metabolic load in a cortical column (Sokoloff et al., 1977), and at least in monkey visual areas there is a strong laminar variability in the spike rate and presumed energy utilization (Snodderly and Gur, 1995). Yet per $\mathrm{mm}^{3}$ (Fig. 12). Last, we find that $70 \%$ of all cells within the sampled regions of cortex are neuronal. This compares with previously reported neuronal fractions of $40 \%$ (Irintchev et al., 2005), 55\% (Herculano-Houzel et al., 2006), 65\% (Ciaroni et al., 1998), and 75\% (Bass et al., 1971).

In comparison with cell counts in other species that range from larger rodents up through monkeys, the neuronal density in mice is $\sim 3 \times$ as great (Fig. 12). In comparison with humans, the neuronal density in mice is $\sim 4 \times$ as great (Fig. 12). However, the total cell count density in mice is only $\sim 1.5 \times$ larger than that found in other species, with considerable variability (Fig. 12). Thus mice have a relatively high ratio of neurons to nonneuronal cells, i.e., $\sim 3: 1$, while the average ratio across other species is $<1: 1$ (Tower and Young, 1973).

\section{Comparison with past vascular measures}

We find that the average vessel diameter of mouse microvessels is $3.5-4.0 \mu \mathrm{m}$. This is consistent with diameters found in in vivo 
measurements (supplemental Fig. 3, available at www.jneurosci. org as supplemental material) and derived from thin tissue sections (Boero et al., 1999) (Fig. 13). We also find an average of $0.88 \mathrm{~m}$ of vasculature length per $\mathrm{mm}^{3}$ of mouse cortex (Fig. 13). This value is consistent with extrapolated values from volumetric data (Heinzer et al., 2008) and that reported from manual traces of thin tissue sections, i.e., $0.7-1.2 \mathrm{~m}$ per $\mathrm{mm}^{3}$ (Boero et al., 1999). Interestingly, the length density of the microvasculature in mice is about twice that in primates (Fig. 13).

The total vasculature volume that we find, $\sim 1 \%$ of the cortical volume, is a factor of two to three smaller than previously reported volumes for mice using imaging techniques (Heinzer et al., 2006, 2008; Serduc et al., 2006) and a factor of 1.5 smaller than that reported using a radio-labeling technique (Zhao and Pollack, 2009). Unlike the measure of vascular length, the total vascular volume further depends on the radii calculated from the vascular data. With regard to image analysis, we attribute our lower measure of vascular volume to the use of local thresholds, which accounts for the point spread function of the optical system, to accurately segment the vascular images (Fig. $7 i, j$ ). The common practice of applying a threshold whose value is close to the noise floor can result in artificially enlarged diameters (supplemental Fig. 4, available at www.jneurosci.org as supplemental material), a criticism that may apply to data for species other than mice (Fig. 13).

\section{Labeling and the technology of complete counting}

An important characteristic about our preparation is the ability to stain and visualize both the vasculature and the surrounding cellular nuclei throughout thick tissue sections. We used DAPI staining to label all cell nuclei, regardless of cellular subtype, since cellular nuclei are more readily segregated than cell somata. We used NeuN antibody staining to classify the cells as neuronal versus non-neuronal and extended deep immunostaining from $500 \mu \mathrm{m}$ sections (Hamam and Kennedy, 2003) to $1400 \mu \mathrm{m} \mathrm{sec}-$ tions (supplemental Fig. 1, available at www.jneurosci.org as supplemental material). Last, we improved upon our previous technique for filling the vasculature (Shih et al., 2009) and developed a gelatin perfusate based on porcine skin gelatin and fluorescein conjugated to albumin. Crosslinking of the albumin to the gelatin skeleton prevents diffusion of the fluorophore, even in exposed vessels.

As a means to visualize the labeled structures deep within the histological tissue preparation, we cleared the tissue by immersion in an index-matching solution of sucrose (Fig. $2 e, g$ ). The use of index-matching solutions to reduce scattering in tissue has a 70 year history (Cumley et al., 1939) and enjoys contemporary use in combination with confocal laser scanning microscopy (CLSM) (Dickie et al., 2006; Wagner et al., 2006). More recently, aggressive clearing techniques have been reported on whole mouse brain (Dodt et al., 2007). We found that the combination of graded sucrose clearing and permeabilization with a detergent effectively minimized tissue shrinkage (Fig. $2 h$ ) and was compatible with immunohistology and imaging with TPLSM.

A suite of programs, coded in the Matlab programming environment, was developed to refine and quantify the image data (Fig. 4; supplemental Information, available at www.jneurosci. org). Adjacent stacks of grayscale image data (Fig. 3) were converted into large binary masks of vessel centerlines (Fig. 6), vessel volume (Fig. 7), cell position (Fig. 5), and cell type (Fig. 8). This refined characterization was used to quantify the cellular and vascular statistics over large tissue volumes. While our algorithms rely on a fairly large number of parameters, the analysis results were found to be sensitive to only a few key parameters (supplemental Tables 1-5, available at www.jneurosci.org as supplemental material). This resulted in the estimate of vascular density, but not cell density, to be dominated by systematic rather than random errors.

The image analyses in the present work extends past methods to create a comprehensive approach for the analysis of both nuclei and vessels in optically sectioned tissue. The closest approximation to our large-scale neuronal analysis comes from recent work by Oberlaender et al. (2009), who count neurons in $\alpha$-NeuN-stained sections from both confocal and two-photon image stacks with voxel sizes that ranged from $0.23 \times 0.23 \times 0.5$ $\mu \mathrm{m}^{3}$ to $0.78 \times 0.78 \times 5.0 \mu \mathrm{m}^{3}$. Their automatic counting method segments neurons based on foreground versus background levels of staining using normalization, median filtering, and morphological operations. The majority of clusters of neurons that touch are separated with a marker-based watershed transformation, while the remainder are separated by a modelbased algorithm that assumes clusters are composed of a small number of neurons whose size is estimated from a histogram over all clusters. Oberlaender et al. (2009) showed that their automated methods were in agreement with manual counts of $\alpha$-NeuN-positive cells; the false-negative rate is $5.9 \pm 3.2 \%$ (average $\pm \mathrm{SD}$ ) and the false-positive rate is $5.0 \pm 2.1 \%$. We argue, however, that the uneven levels of $\alpha$-NeuN staining (Fig. 3h) could result in undercounts in both automated and manual estimates, and that our use of DAPI provides a relatively unambiguous measure to detect a cell prior to classification as a neuron based on $\alpha$-NeuN staining (Fig. 8c).

The closest approximation to the vessel analyses presented here are found in the work of Cassot and colleagues (Fouard et al., 2004; Cassot et al., 2006) and Soltanian-Zadeh and colleagues (Maddah et al., 2003; 2005), who used CLSM to optically section preparations in which vessels were filled with a contrast agent. A critical improvement is our use of the point-spread function in the estimation of vessel diameter (Fig. 7h,i; supplemental Fig. 4, available at www.jneurosci.org as supplemental material). With regards to segmentation, Kirbas and Quek (2004) review the state-of-the-art and define six broad areas of vessel extraction procedures: (1) pattern recognition techniques, which includes matched filtering; (2) model-based approaches; (3) tracking-based approaches; (4) artificial intelligence-based approaches; (5) neural network-based approaches; and (6) tube-like object detection approaches. The three-dimensional vessel segmentation methods used here are an extension of the two-dimensional matched filtering methods.

\section{Epilog}

The immunohistological staining techniques presented in this work to label and clear thick slabs of cortex, together with the analysis algorithms developed to extract and quantify the topology of the labeled structures, are compatible with automated block-face imaging (Tsai et al., 2003; McCormick et al., 2004; Ragan et al., 2007). The reconstruction of volumes that span entire mouse brains will impose additional needs for algorithmic development, such as gap-filling routines to connect broken strands of vasculature across separately imaged blocks of tissues (Kaufhold et al., 2008; Risser et al., 2008). Nonetheless, we suggest that the present work provides a means to gain an accurate statistical analysis of cortical anatomy by measuring all cells and vessels and an understanding of vascular topology for simulation of blood flow in cortex (Boas et al., 2008; Reichold et al., 2009; Ress et al., 2009). 


\section{References}

Abreu-Villaça Y, Silva WC, Manhães AC, Schmidt SL (2002) The effect of corpus callosum agenesis on neocortical thickness and neuronal density of BALB/cCF mice. Brain Res Bull 58:411-416.

Bass NH, Hess HH, Pope A, Thalheimer C (1971) Quantitative cytoarchitectonic distribution of neurons, glia, and $\mathrm{DNa}$ in rat cerebral cortex. J Comp Neurol 143:481-490.

Bell MA, Ball MJ (1985) Laminar variation in the microvascular architecture of normal human visual cortex (area 17). Brain Res 335:139-143.

Bjornsson CS, Lin G, Al-Kofahi Y, Narayanaswamy A, Smith KL, Shain W, Roysam B (2008) Associative image analysis: A method for automated quantification of $3 \mathrm{D}$ multi-parameter images of brain tissue. J Neurosci Methods 170:165-178.

Boas DA, Jones SR, Devor A, Huppert TJ, Dale AM (2008) A vascular anatomical network model of the spatio-temporal response to brain activation. Neuroimage 40:1116-1129.

Boero JA, Ascher J, Arregui A, Rovainen C, Woolsey TA (1999) Increased brain capillaries in chronic hypoxia. J Appl Physiol 86:1211-1219.

Bonthius DJ, McKim R, Koele L, Harb H, Karacay B, Mahoney J, Pantazis NJ (2004) Use of frozen sections to determine neuronal number in the murine hippocampus and neocortex using the optical disector and optical fractionator. Brain Res Protoc 14:45-57.

Brunjes PC (1985) A stereological study of neocortical maturation in the precocial mouse, Acomys cahirinus. Brain Res 351:279-287.

Carmeliet P, Tessier-Lavigne M (2005) Common mechanisms of nerve and blood vessel wiring. Nature 436:193-200.

Cassot F, Lauwers F, Fouard C, Prohaska S, Lauwers-Cances V (2006) A novel three-dimensional computer-assisted method for a quantitative study of microvascular networks of the human cerebral cortex. Microcirculation 13:1-18.

Cavaglia M, Dombrowski SM, Drazba J, Vasanji A, Bokesch PM, Janigro D (2001) Regional varition in brain capillary density and vascular response to ishemia. Brain Res 910:81-93.

Chow K, Blum JS, Blum RA (1950) Cell ratios in the thalamo-cortical visual system of Macaca mulatta. J Comp Neurol 92:227-239.

Ciaroni S, Buffi O, Ambrogini P, Cecchini T, Del Grande P (1998) Neuron and glial cells in neocortex after methylazoxymethanol treatment in early development. Mech Ageing Dev 100:299-311.

Cowey A (1964) Projection of the retina on to striate and prestriate cortex in the squirrel monkey, Saimiri sciureus. J Neurophysiol 27:366-393.

Cragg BG (1967) The density of synapses and neurones in the motor and visual areas of the cerebral cortex. J Anat 101:639-654.

Cumley RW, Crow JF, Griffen AB (1939) Clearing specimens for the demonstration of bone. Stain Technol 14:7-11.

Dickie R, Bachoo RM, Rupnick MA, Dallabrida SM, DeLoid M, Lai J, DePinho RA, Dickie R, Rogers RA (2006) Three-dimensional visualization of microvessel architecture of whole-mount tissue by confocal microscopy. Microvasc Res 72:20-26.

Dodt HU, Leischner U, Schierloh A, Jährling N, Mauch CP, Deininger K, Deussing JM, Eder M, Zieglgänsberger W, Becker K (2007) Ultramicroscopy: three-dimensional visualization of neuronal networks in the whole mouse brain. Nat Meth 4:331-336.

Dutly AE, Kugathasan L, Trogadis JE, Keshavjee SH, Stewart DJ, Courtman DW (2006) Fluorescent microangiography (FMA): an improved tool to visualize the pulmonary microvasculature. Lab Invest 86:409-416.

Fouard C, Malandain G, Prohaska S, Westerhoff M, Cassot F, Mazel C, Asselot D, Marc-Vergnes JP (2004) Skeletonization by blocks for large datasets: application to brain microcirculation. Paper presented at the International Symposium on Biomedical Imaging: From Nano to Macro, Arlington, VA, April.

Fukui Y, Hayasaka S, Itoh M, Takeuchi Y (1992) Development of neurons and synapses in ochratoxin a-induced microcephalic mice: a quantitative assessment of somatosensory cortex. Neurotoxicol Teratol 14:191-196.

Garey LJ, Leuba G (1986) A quantitative study of neuronal and glial numerical density in the visual cortex of the bottlenose dolphin: evidence for a specialized subarea and changes with age. J Comp Neurol 247:491-496.

Gittins R, Harrison PJ (2004) Neuronal density, size and shape in the human anterior cingulate cortex: a comparison of Nissl and NeuN staining. Brain Res Bull 63:155-160.

Gredal O, Pakkenberg H, Karlsborg M, Pakkenberg B (2000) Unchanged total number of neurons in motor cortex and neocortex in amyotrophic lateral sclerosis: a stereological study. J Neurosci Methods 95:171-176.
Hamam BN, Kennedy TE (2003) Visualization of the dendritic arbor of neurons in intact 500 microm thick brain slices. J Neurosci Methods 123:61-67.

Harper C, Kril J (1989) Patterns of neuronal loss in the cerebral cortex in chronic alcoholic patients. J Neurol Sci 92:81-89.

Harrison RV, Harel N, Panesar J, Mount RJ (2002) Blood capillary distribution correlates with hemodynamic-based functional imaging in cerebral cortex. Cereb Cortex 12:225-233.

Heinzer S, Krucker T, Stampanoni M, Abela R, Meyer EP, Schuler A, Schneider P, Müller R (2006) Hierarchical microimaging for multiscale analysis of large vascular networks. Neuroimage 32:626-636.

Heinzer S, Kuhn G, Krucker T, Meyer E, Ulmann-Schuler A, Stampanoni M, Gassmann M, Marti HH, Müller R, Vogel J (2008) Novel threedimensional analysis tool for vascular trees indicates complete micronetworks, not single capillaries, as the angiogenic endpoint in mice overexpressing human $\operatorname{VEGF}(165)$ in the brain. Neuroimage 39: 1549-1558.

Herculano-Houzel S, Mota B, Lent R (2006) Cellular scaling rules for rodent brains. Proc Natl Acad Sci U S A 103:12138-12143.

Herculano-Houzel S, Collins CE, Wong P, Kaas JH (2007) Cellular scaling rules for primate brains. Proc Natl Acad Sci U S A 104:3562-3567.

Hodge RD, D'Ercole AJ, O'Kusky JR (2005) Increased expression of insulinlike growth factor-I (IGF-I) during embryonic development produces neocortical overgrowth with differentially greater effects on specific cytoarchitectonic areas and cortical layers. Brain Res Dev Brain Res 154:227-237.

Irintchev A, Rollenhagen A, Troncoso E, Kiss JZ, Schachner M (2005) Structural and functional aberrations in the cerebral cortex of tenascin-C deficient mice. Cereb Cortex 15:950-962.

Kaufhold J, Tsai PS, Blinder P, Kleinfeld D (2008) Threshold relaxation is an effective means to connect gaps in 3D images of complex microvascular networks. Paper presented at the Third Workshop on Microscopic Image Analysis with Applications in Biology: MICCAI, New York, September.

Kaufhold JP, Tsai PS, Blinder P, Friedman B, Lyden PD, Kleinfeld D, Ifarraguerri AI (2007) Automated neuroanatomical microvessel segmentation, nuclei labeling and geometric computations with $3 \mathrm{D}$ histological data. Soc Neurosci Abstr 33:87.14/PP3.

Kirbas C, Quek F (2004) A review of vessel extraction techniques and algorithms. Ass Comp Mach Comp Surv 36:81-121.

Kril JJ, Harper CG (1989) Neuronal counts from four cortical regions of alcoholic brains. Acta Neuropathologica 79:200-204.

Krucker T, Lang A, Meyer EP (2006) New polyurethane-based material for vascular corrosion casting with improved physical and imaging characteristics. Microsc Res Tech 69:138-147.

Lauwers F, Cassot F, Lauwers-Cances V, Puwanarajah P, Duvernoy H (2008) Morphometry of the human cerebral cortex microcirculation: general characteristics and space-related profiles. Neuroimage 39:936-948.

Lecoq J, Tiret P, Najac M, Shepherd GM, Greer CA, Charpak S (2009) Odorevoked oxygen consumption by action potential and synaptic transmission in the olfactory bulb. J Neurosci 29:1424-1433.

Lefort S, Tomm C, Floyd Sarria JC, Petersen CC (2009) The excitatory neuronal network of the $\mathrm{C} 2$ barrel column in mouse primary somatosensory cortex. Neuron 61:301-316.

Leuba G, Rabinowicz T (1979) Long-term effects of postnatal undernutrition and maternal malnutrition on mouse cerebral cortex I. Cellular densities, cortical volume and total numbers of cells. Exp Brain Res 37:283-298.

Logothetis NK, Pauls J, Augath M, Trinath T, Oeltermann A (2001) Neurophysiological investigation of the basis of the fMRI signal. Nature 412:150-157.

Lok J, Gupta P, Guo S, Kim WJ, Whalen MJ, van Leyen K, Lo EH (2007) Cell-cell signaling in the neurovascular unit. Neurochem Res 32:2032-2045.

Lyck L, Krøigård T, Finsen B (2007) Unbiased cell quantification reveals a continued increase in the number of neocortical neurones during early post-natal development in mice. Eur J Neurosci 26:1749-1764.

Ma D, Descarries L, Micheva KD, Lepage Y, Julien JP, Doucet G (1999) Severe neuronal losses with age in the parietal cortex and ventrobasal thalamus of mice transgenic for the human NF-L neurofilament protein. J Comp Neurol 406:433-448.

Maddah M, Afzali-Kusha A, Soltanian-Zadeh H (2003) Efficient center-line 
extraction for quantification of vessels in confocal microscopy images. Med Phys 30:204-211.

Maddah M, Soltanian-Zadeh H, Afzali-Kusha A, Shahrokni A, Zhang ZG (2005) Three-dimensional analysis of complex branching vessels in confocal microscopy images. Comput Med Imaging Graph 29:487-498.

McCormick BH, Koh W, Choe Y, Abbott LC, Keyser J, Mayerich D, Melek Z, Doddapaneni P (2004) Construction of anatomically correct models of mouse brain networks. Neurocomputing 58-60:379-386.

Michaloudi H, Grivas I, Batzios C, Chiotelli M, Papadopoulos GC (2005) Areal and laminar variations in the vascularity of the visual, auditory, and entorhinal cortices of the developing rat brain. Dev Brain Res 155:60-70.

Motti ED, Imhof HG, Yaşargil MG (1986) The terminal vasculature bed in the superficial cortex of the rat: an SEM study of corrosion casts. J Neurosurg 65:834-846.

Mountjoy CQ, Roth M, Evans NJ, Evans HM (1983) Cortical neuronal counts in normal elderly controls and demented patients. Neurobiol Aging 4:1-11.

Nguyen QT, Tsai PS, Kleinfeld D (2006) MPScope: A versatile software suite for multiphoton microscopy. J Neurosci Methods 156:351-359.

Nguyen QT, Dolnick EM, Driscoll J, Kleinfeld D (2009) MPScope 2.0: a computer system for two-photon laser scanning microscopy with concurrent plasma-mediated ablation and electrophysiology. In: Methods for in vivo optical imaging, Ed 2 (Frostig RD, ed), pp 117-142. Boca Raton, FL: CRC.

Oberlaender M, Dercksen VJ, Egger R, Gensel M, Sakmann B, Hege HC (2009) Automated three-dimensional detection and counting of neuron somata. J Neurosci Methods 180:147-160.

O'Kusky J, Colonnier M (1982) A laminar analysis of the number of neurons, glia, and synapses in the visual cortex (Area 17) of adult macaque monkeys. J Comp Neurol 210:278-290.

Pakkenberg B, Gundersen HJ (1997) Neocortical neuron number in humans: effect of sex and age. J Comp Neurol 384:312-320.

Patel U (1983) Non-random distribution of blood vessels in the posterior region of the rat somatosensory cortex. Brain Res 289:65-70.

Ragan T, Sylvan JD, Kim KH, Huang H, Bahlmann K, Lee RT, So PT (2007) High-resolution whole organ imaging using two-photon tissue cytometry. J Biomed Opt 12:014015.

Ramon-Moliner E (1961) The histology of the post-cruciate gyrus in the cat. I. Quantitative studies. J Comp Neurol 117:43-62.

Regeur L, Jensen GB, Pakkenberg H, Evans SM, Pakkenberg B (1994) No global neocortical nerve cell loss in brains from patients with senile dementia of Alzheimer's type. Neurobiol Aging 15:347-352.

Reichold J, Stampanoni M, Lena Keller A, Buck A, Jenny P, Weber B (2009) Vascular graph model to simulate the cerebral blood flow in realistic vascular networks. J Cereb Blood Flow Metab 29:1429-1443.

Ress D, Thompson JK, Rokers B, Khan RK, Huk AC (2009) A model for transient oxygen delivery in cerebral cortex. Front Neuroenergetics 1:3.

Risser L, Plouraboué F, Cloetens P, Fonta C (2009) A 3D-investigation shows that angiogenesis in primate cerebral cortex mainly occurs at capillary level. Int J Dev Neurosci 27:185-196.

Risser L, Plouraboue F, Descombes X (2008) Gap filling of 3-D microvascular networks by tensor voting. IEEE Trans Med Imaging 27:674-687.

Rousselle CH, Lefauconnier JM, Allen DD (1998) Evaluation of anesthetic effects on parameters for the in situ rat brain perfusion technique. Neurosci Lett 257:139-142.

Russ JC, Dehoff RT (2000) Practical stereology, Ed 2. New York: Plenum.

Schlageter KE, Molnar P, Lapin GD, Groothuis DR (1999) Microvessel organization and structure in experimental brain tumors: microvessel populations with distinctive structural and functional properties. Microvasc Res 58:312-328.

Schüz A, Palm G (1989) Density of neurons and synapses in the cerebralcortex of the mouse. J Comp Neurol 286:442-455.

Serduc R, Vérant P, Vial JC, Farion R, Rocas L, Rémy C, Fadlallah T, Brauer E, Bravin A, Laissue J, Blattmann H, van der Sanden B (2006) In vivo twophoton microscopy study of short-term effects of microbeam irradiation on normal mouse brain microvasculature. Int J Radiat Oncol Biol Phys 64:1519-1527.
Shih AY, Friedman B, Drew PJ, Tsai PS, Lyden PD, Kleinfeld D (2009) Active dilation of penetrating arterioles restores red blood cell flux to penumbral neocortex after focal stroke. J Cereb Blood Flow Metab 29:738-751.

Sholl DA (1959) A comparative study of the neuronal packing density in the cerebral cortex. J Anat 93:143-158

Silva AC, Koretsky AP (2002) Laminar specificity of functional MRI onset times during somatosensory stimulation in rat. Proc Natl Acad Sci U S A 99:15182-15187.

Snodderly DM, Gur M (1995) Organization of striate cortex of alert, Tained monkeys (Macaca fascicularis): ongoing activity, stimulus selectivity, and widths of receptive field activating regions. J Neurophysiol 74:2100-2125.

Sokoloff L, Reivich M, Kennedy C, Des Rosiers MH, Patlak CS, Pettigrew KD, Sakurada O, Shinohara M (1977) he [14C]deoxyglucose method for the measurement of local cerebral glucose utilization: theory, procedure, and normal values in the conscious and anesthetized albino rat. J Neurochem 28:897-916.

Stepnoski RA, LaPorta A, Raccuia-Behling F, Blonder GE, Slusher RE, Kleinfeld D (1991) Noninvasive detection of changes in membrane potential in cultured neurons by light scattering. Proc Natl Acad Sci U S A 88:9382-9386.

Stewart PA, Isaacs H, LaManna JC, Harik SI (1997) Ultrastructural concomitants of hypoxia-induced angiogenesis. Acta Neuropathol 93:579584 .

Stubbs D, DeProto J, Nie K, Englund C, Mahmud I, Hevner R, Molnár Z (2009) Neurovascular congruence during cerebral cortical development. Cereb Cortex 19:32-41.

Tieman SB, Möllers S, Tieman DG, White JT (2004) The blood supply of the cat's visual cortex and its postnatal development. Brain Res 998:100-112.

Tower DB, Elliot KAC (1952) Structural and functional organisation of mammalian cerebral cortex: the correlations of neurone density with brain size. J Comp Neurol 85:313-420 .

Tower DB, Young OM (1973) The activities of butyrylcholinesterase and carbonic anhydrase, the rate of anaerobic glycolysis, and the question of a constant density of glial cells in cerebral cortices of various mammalian species from mouse to whale. J Neurochem 20:269-278.

Tsai PS, Kleinfeld D (2009) In vivo two-photon laser scanning microscopy with concurrent plasma-mediated ablation: Principles and hardware realization. In: Methods for in vivo optical imaging, Ed 2 (Frostig RD, ed), pp 59-115. Boca Raton, FL: CRC.

Tsai PS, Friedman B, Ifarraguerri AI, Thompson BD, Lev-Ram V, Schaffer CB, Xiong Q, Tsien RY, Squier JA, Kleinfeld D (2003) All-optical histology using ultrashort laser pulses. Neuron 39:27-41.

Tsai PS, Kaufhold JP, Friedman B, Ifarraguerri AI, Lyden PD, Kleinfeld D (2007) Direct counts of cellular and vascular densities in thick cortical sections of mouse. Soc Neurosci Abstr 33:87.12/PP1.

Vérant P, Serduc R, van der Sanden B, Rémy C, Vial JC (2007) A direct method for measuring mouse capillary cortical blood volume using multiphoton laser scanning microscopy. J Cereb Blood Flow Metab 27:10721081.

Wagner RC, Czymmek K, Hossler FE (2006) Confocal microscopy, computer modeling, and quantification of glomerular vascular corrosion casts. Microsc Microanal 12:262-268.

Weber B, Keller AL, Reichold J, Logothetis NK (2008) The microvascular system of the striate and extrastriate visual cortex of the macaque. Cereb Cortex 18:2318-2330.

Witelson SF, Glezer II, Kigar DL (1995) Women have greater density of neurons in posterior temporal cortex. J Neurosci 15:3418-3428.

Zhang S, Murphy TH (2007) Imaging the impact of cortical microcirculation on synaptic structure and sensory-evoked hemodynamic responses in vivo. PLoS Biol 5:e119.

Zhao R, Pollack GM (2009) Regional differences in capillary density, perfusion rate, and P-glycoprotein activity: a quantitative analysis of regional drug exposure in the brain. Biochem Pharmacol 78:1052-1059.

Zheng D, LaMantia AS, Purves D (1991) Specialized vascularization of the primate visual cortex. J Neurosci 11:2622-2629. 